\title{
Phase structure, collective modes, and the axial anomaly in dense QCD
}

\author{
Naoki Yamamoto, ${ }^{1}$ Motoi Tachibana, ${ }^{2}$ Tetsuo Hatsuda, ${ }^{1}$ and Gordon Baym ${ }^{3}$ \\ ${ }^{1}$ Department of Physics, University of Tokyo, Japan \\ ${ }^{2}$ Department of Physics, Saga University, Saga 840-8502, Japan \\ ${ }^{3}$ Department of Physics, University of Illinois, 1110 W. Green St., Urbana, Illinois 61801
}

\begin{abstract}
Using a general Ginzburg-Landau effective Lagrangian, we study the topological structure and low-lying collective modes of dense QCD having both chiral and diquark condensates, for two and three massless flavors. As we found earlier, the QCD axial anomaly acts as an external field applied to the chiral condensate in a color superconductor and, as a new critical point emerges, leads to a crossover between the broken chiral symmetry and color superconducting phases. At intermediate densities where both chiral and diquark condensates are present, we derive a generalized Gell-MannOakes-Renner relation between the masses of pseudoscalar bosons and the magnitude of the chiral and diquark-condensates. We show explicitly the continuity of the ordinary pion at low densities to a generalized pion at high densities.
\end{abstract}

PACS numbers: 12.38.-t,12.38.Mh,26.60.+c

\section{INTRODUCTION}

Quantum chromodynamics at finite temperature, $T$, and chemical potential, $\mu$, has a rich phase structure: at low $T$ and $\mu$, the Nambu-Goldstone (NG) phase with nearly massless pions is realized by the dynamical breaking of chiral symmetry through condensation of quark-anti-quark pairs [1, 2]. On the other hand, at low $T$ and high $\mu$, a Fermi liquid of deconfined quarks is expected to appear as a consequence of asymptotic freedom [3]. Furthermore, in cold quark matter, condensation of quark-quark pairs [4, 5] leads to a color superconducting phase (CSC) and dynamical breaking of color gauge symmetry. At high $T$ for any $\mu$, the condensates melt away and a quark-gluon plasma symmetric under $S U(3)_{C}$ is realized [ $[6]$. The experimental exploration of quark-gluon plasmas is being actively carried out in ultrarelativistic heavy ion collisions at RHIC, and will be continued in the future at the LHC. The transition from the NG phase to the CSC is also relevant to heavy ion collisions to be realized in the future at moderate energies at GSI, and is of interest in the interiors of neutron stars and possible quark stars.

We focus our attention here on the QCD phase structure at intermediate densities and study the important interplay between two competing phenomena: quark-anti-quark pairing, characterized by a chiral condensate $\Phi \sim\langle\bar{q} q\rangle$, and quark-quark pairing, characterized by a diquark condensate $d \sim\langle q q\rangle$. This interplay is interesting not only in its own right, but also in relation to similar phenomena in other systems, e.g., the interplay between magnetically ordered phases and metallic superconductivity [7]. Many works on the phase structure in the intermediate density region have been carried out using effective models such as that of Nambu and Jona-Lasinio (NJL) [8] and the random matrix model [9]. In Ref. [10] we introduced a model-independent approach to construct a general Ginzburg-Landau (GL) effective Lagrangian in terms of the two order parameter fields, $\Phi$ and $d$, and showed, in particular, that the $\Phi$ - $d$ coupling induced by the axial anomaly leads to a new critical point in the QCD phase diagram, implying a crossover between the NG and CSC phases. The crossover is relevant to the question of "hadron-quark continuity" [11].

The purposes of this paper are twofold: Firstly, we give a detailed account of the role of the axial anomaly in creating the topological structure of the QCD phase diagram discussed in Ref. [10]. Secondly, we study the properties of the collective modes, the pseudoscalar nonet $\left(\pi, K, \eta, \eta^{\prime}\right)$ and the $H$ (the NG boson associated with the breaking of $U(1)$ baryon symmetry), in the intermediate density regime where both chiral and diquark condensates are present.

This paper is organized as follows. In Sec. III, we construct a general GL free-energy in terms of the chiral and diquark fields that is invariant under global chiral and local color symmetry. We then use the GL free energy to classify possible phases for two characteristic cases: three-flavor quark matter with equal numbers of massless up, down and strange quarks, and two-flavor quark matter with equal numbers of massless up and down quarks; and then conjecture the phase diagram for realistic quark masses (light up and down quarks and a medium-heavy strange quark) from the results of the two cases. In Sec. III] after showing the essential features of the mixing between independent massless modes by a toy model, we build an effective Lagrangian for the "pions" $(\pi, K, \eta)$, and the $H$ boson, and derive a generalized Gell-Mann-Oakes-Renner (GOR) relation in dense QCD between the masses of the pseudo-scalar bosons and the magnitude of the chiral and diquark-condensates. We discuss the continuity of the NG phase and CSC phase in the excited states. Section[V] is devoted to summary and conclusion. In appendices, we give the detailed derivation of the phase diagrams, magnitudes and signs of the GL free-energy parameters, and the mass spectra of $\eta^{\prime}$ mesons. 


\section{PHASE STRUCTURE}

In this section, we construct, on the basis of QCD symmetry, the most general GL free-energy for the chiral field, the diquark fields and their mutual couplings in three spatial dimensions. We then classify the possible phase structures of massless QCD with two and three flavors, and discuss the QCD phase structure in the real world.

\section{A. Ginzburg-Landau free energy}

We write the general GL free energy, measured with respect to that of the normal phase, $\Phi=d_{L}=d_{R}=0$, as

$$
\Omega\left(\Phi, d_{L}, d_{R}\right)=\Omega_{\chi}(\Phi)+\Omega_{d}\left(d_{L}, d_{R}\right)+\Omega_{\chi d}\left(\Phi, d_{L}, d_{R}\right)
$$

where $\Phi$, the chiral condensate, and $d_{L, R}$, the diquark condensates, are the basic order parameter fields; $\Omega_{\chi d}$ denotes the coupling between these order parameters. The form of $\Omega\left(\Phi, d_{L}, d_{R}\right)$ is severely constrained by the QCD symmetry ${ }^{1}$

$$
\mathcal{G} \equiv S U(3)_{L} \times S U(3)_{R} \times U(1)_{B} \times U(1)_{A} \times S U(3)_{C}
$$

The axial anomaly breaks the $U(1)_{A}$ symmetry down to $Z\left(2 N_{f}\right)_{A}=Z(6)_{A}$ where $N_{f}$ is the number of massless flavors. Under $\mathcal{G}$, the left and right handed quarks transform as

$$
q_{L} \rightarrow \mathrm{e}^{-i \alpha_{A}} \mathrm{e}^{-i \alpha_{B}} V_{L} V_{C} q_{L}, \quad q_{R} \rightarrow \mathrm{e}^{i \alpha_{A}} \mathrm{e}^{-i \alpha_{B}} V_{R} V_{C} q_{R}
$$

where $V_{L, R, C}$ are $S U(3)_{L, R, C}$ rotations with continuous parameters and the phases $\alpha_{A, B}$ are associated with $U(1)_{A, B}$ rotations.

We introduce the chiral fields $\Phi_{i j}$ (with $i$ and $j$ flavor indices) of mass dimension one and singlets under $S U(3){ }_{C}$ and $U(1)_{B}$, as

$$
\Phi_{i j}=G_{\chi}\left\langle\bar{q}_{R a}^{j} q_{L a}^{i}\right\rangle
$$

The precise proportionality constant, $G_{\chi}$, enters only in numerical evaluation of the GL free energy parameters; see Appendix B. The chiral fields transform under $\mathcal{G}$ as

$$
\Phi \rightarrow \mathrm{e}^{-2 i \alpha_{A}} V_{L} \Phi V_{R}^{\dagger}
$$

In addition,

$$
\Phi \Phi^{\dagger} \rightarrow V_{L}\left(\Phi \Phi^{\dagger}\right) V_{L}^{\dagger}, \quad \Phi^{\dagger} \Phi \rightarrow V_{R}\left(\Phi^{\dagger} \Phi\right) V_{R}^{\dagger}, \quad \operatorname{det} \Phi \rightarrow \mathrm{e}^{-6 i \alpha_{A}} \operatorname{det} \Phi .
$$

Note the invariances of $\Phi$ under $Z(2)_{A} \subset U(1)_{A}$, and det $\Phi$ under $Z(6)_{A} \subset U(1)_{A}$.

The most general form of the free energy of the chiral field, up to $\mathcal{O}\left(\Phi^{4}\right)$, that is consistent with these transformations reads [12],

$$
\Omega_{\chi}=\frac{a_{0}}{2} \operatorname{Tr} \Phi^{\dagger} \Phi+\frac{b_{1}}{4 !}\left(\operatorname{Tr} \Phi^{\dagger} \Phi\right)^{2}+\frac{b_{2}}{4 !} \operatorname{Tr}\left(\Phi^{\dagger} \Phi\right)^{2}-\frac{c_{0}}{2}\left(\operatorname{det} \Phi+\operatorname{det} \Phi^{\dagger}\right)
$$

where "Tr" and "det" are taken over the flavor indices, $i$ and $j$. The first three terms on the right side of Eq. (7) are invariant under the full symmetry group $\mathcal{G}$, while the fourth term, caused by the axial anomaly, breaks the $U(1)_{A}$ symmetry down to $Z(6)_{A}$. The free energy $\Omega_{\chi}$ is bounded below for large values of the condensates for $b_{1}+b_{2} / 3>0$ and $b_{2}>0$. If these conditions are not met, we need to introduce terms $\mathcal{O}\left(\Phi^{6}\right)$ to stabilize the free energy, a situation we will encounter later. We assume $c_{0}$ to be positive so that the condensate at low temperature is positive, while simultaneously the $\eta^{\prime}$ mass obeys $m_{\eta^{\prime}}^{2}>0$. We assume that $a_{0}$ changes sign at a certain temperature to drive the chiral phase transition.

\footnotetext{
1 More precisely, to avoid double counting the $Z(3)$ center in $S U(3)$ and the discrete subgroup of $U(1)$, the chiral $S U(3)_{L, R}$ 's in Eq. (2) are the quotient groups, $S U(3)_{L, R} / Z(3)$.
} 
We define the Lorentz-scalar diquark order parameters,

$$
G_{d}\left\langle q_{L b}^{j} C q_{L c}^{k}\right\rangle=\epsilon_{a b c} \epsilon_{i j k}\left[d_{L}^{\dagger}\right]_{a i}, \quad G_{d}\left\langle q_{R b}^{j} C q_{R c}^{k}\right\rangle=\epsilon_{a b c} \epsilon_{i j k}\left[d_{R}^{\dagger}\right]_{a i},
$$

where $i, j, k$ are the flavor and $a, b, c$ the color indices; $C=i \gamma^{2} \gamma^{0}$ is the charge conjugation matrix with the properties, $C C^{\dagger}=-C^{2}=1$ and $\gamma^{0} C^{\dagger} \gamma^{0}=C$. The $3 \times 3$ matrix $\left[d_{L, R}\right]_{i a}$ belongs to the fundamental representation of $S U(3)_{C} \times$ $S U(3)_{L, R}$. The positive proportionality constant $G_{d}$, is introduced to make the mass dimension of $d_{L, R}$ one; it enters only in numerical evaluation of the GL free energy parameters (see Appendix Cl). The transformation properties of the $d$ 's under $\mathcal{G}$ are

$$
d_{L} \rightarrow e^{2 i \alpha_{A}} e^{2 i \alpha_{B}} V_{L} d_{L} V_{C}^{\mathrm{T}}, \quad d_{R} \rightarrow e^{-2 i \alpha_{A}} e^{2 i \alpha_{B}} V_{R} d_{R} V_{C}^{\mathrm{T}}
$$

Note that $d_{L(R)}$ is invariant under $S U(3)_{R(L)}$, and $Z(2)_{B-A(B+A)}$. Also the color-singlet combinations of the diquark fields transform as

$$
\begin{aligned}
d_{L} d_{L}^{\dagger} & \rightarrow V_{L}\left(d_{L} d_{L}^{\dagger}\right) V_{L}^{\dagger}, & d_{R} d_{R}^{\dagger} & \rightarrow V_{R}\left(d_{R} d_{R}^{\dagger}\right) V_{R}^{\dagger}, \\
d_{L} d_{R}^{\dagger} & \rightarrow e^{4 i \alpha_{A}} V_{L}\left(d_{L} d_{R}^{\dagger}\right) V_{R}^{\dagger}, & d_{R} d_{L}^{\dagger} & \rightarrow e^{-4 i \alpha_{A}} V_{R}\left(d_{R} d_{L}^{\dagger}\right) V_{L}^{\dagger}, \\
\operatorname{det} d_{L} & \rightarrow e^{6 i \alpha_{A}} e^{6 i \alpha_{B}} \operatorname{det} d_{L}, & \operatorname{det} d_{R} & \rightarrow e^{-6 i \alpha_{A}} e^{6 i \alpha_{B}} \operatorname{det} d_{R}
\end{aligned}
$$

Note that in addition to invariances under $S U(3)_{C}, U(1)_{A}$ and $U(1)_{B}$, the products $d_{L} d_{L}^{\dagger}$ and $d_{R} d_{R}^{\dagger}$ are invariant under $S U(3)_{R}$ and $S U(3)_{L}$ respectively. Furthermore, in addition to invariances under $S U(3)_{C}$, the products $d_{L} d_{R}^{\dagger}$ and $d_{R} d_{L}^{\dagger}$ are invariant under $U(1)_{B}$ and $Z(4)_{A} \subset U(1)_{A}$, while $\operatorname{det} d_{L}$ and $\operatorname{det} d_{R}$ are invariant under $S U(3)_{C}$, $Z(6)_{B} \subset U(1)_{B}$, and $Z(6)_{A} \subset U(1)_{A}$.

The most general form of the GL free energy of the $d$-fields invariant under $\mathcal{G}$ up to $\mathcal{O}\left(d^{4}\right)$ is [13, 14, 15]:

$$
\begin{aligned}
\Omega_{d} & =\alpha_{0} \operatorname{Tr}\left[d_{L} d_{L}^{\dagger}+d_{R} d_{R}^{\dagger}\right]+\beta_{1}\left(\left[\operatorname{Tr}\left(d_{L} d_{L}^{\dagger}\right)\right]^{2}+\left[\operatorname{Tr}\left(d_{R} d_{R}^{\dagger}\right)\right]^{2}\right) \\
& +\beta_{2}\left(\operatorname{Tr}\left[\left(d_{L} d_{L}^{\dagger}\right)^{2}\right]+\operatorname{Tr}\left[\left(d_{R} d_{R}^{\dagger}\right)^{2}\right]\right)+\beta_{3} \operatorname{Tr}\left[\left(d_{R} d_{L}^{\dagger}\right)\left(d_{L} d_{R}^{\dagger}\right)\right]+\beta_{4} \operatorname{Tr}\left(d_{L} d_{L}^{\dagger}\right) \operatorname{Tr}\left(d_{R} d_{R}^{\dagger}\right)
\end{aligned}
$$

The transition from the normal state to color superconductivity is driven by $\alpha_{0}$ changing sign. Unlike for $\Phi$, terms such as det $d_{L, R}$ are not allowed, since $d_{L, R}$ carries baryon number.

Finally, the interaction free-energy of the chiral and diquark fields to fourth order reads [10]

$$
\begin{aligned}
\Omega_{\chi d}= & \gamma_{1} \operatorname{Tr}\left[\left(d_{R} d_{L}^{\dagger}\right) \Phi+\left(d_{L} d_{R}^{\dagger}\right) \Phi^{\dagger}\right]+\lambda_{1} \operatorname{Tr}\left[\left(d_{L} d_{L}^{\dagger}\right) \Phi \Phi^{\dagger}+\left(d_{R} d_{R}^{\dagger}\right) \Phi^{\dagger} \Phi\right] \\
& +\lambda_{2} \operatorname{Tr}\left[d_{L} d_{L}^{\dagger}+d_{R} d_{R}^{\dagger}\right] \cdot \operatorname{Tr}\left[\Phi^{\dagger} \Phi\right]+\lambda_{3}\left(\operatorname{det} \Phi \cdot \operatorname{Tr}\left[\left(d_{L} d_{R}^{\dagger}\right) \Phi^{-1}\right]+h . c\right)
\end{aligned}
$$

The triple boson coupling $\sim \gamma_{1}$, which breaks the $U(1)_{A}$ symmetry down to $Z(6)_{A}$, originates from the axial anomaly. The remaining terms are fully invariant under $\mathcal{G}$. The $\lambda_{3}$ term is equivalent to the polynomial structure, $\epsilon_{i j k} \epsilon_{i^{\prime} j^{\prime} k^{\prime}} \Phi_{i i^{\prime}} \Phi_{j j^{\prime}}\left(d_{L} d_{R}^{\dagger}\right)_{k k^{\prime}}[16]$.

Equations (7), (13) and (14) constitute the most general form of the GL free energy under the conditions that the phase transition is not strongly first order (i.e., the values of $\Phi, d_{L, R}$ are sufficiently smaller than those at zero temperature) and that the condensed phases are spatially homogeneous.

\section{B. Three massless flavors}

We first study three massless flavors. To proceed analytically, we restrict ourselves to maximally symmetric condensates, namely, a flavor symmetric chiral condensate in which quark-anti-quark pairing takes place only within the same flavor and a color-flavor-locked (CFL) diquark condensate in which the quark-quark pairing takes place only in the different flavors:

$$
\begin{aligned}
\Phi & =\operatorname{diag}(\sigma, \sigma, \sigma), \\
d_{L} & =-d_{R}=\operatorname{diag}(d, d, d),
\end{aligned}
$$

where $\sigma$ and $d$ are assumed to be real. We have chosen the relative sign between $d_{L}$ and $d_{R}$ in Eq. (16) corresponding to the ground state having positive parity, as is indeed favored by the axial anomaly together with finite quark masses 
[17]. The CFL condensate in Eq. (16) is indeed realized at asymptotically high density, as shown in [13] via the GL approach, and is the simplest ansatz at intermediate density. Thus the reduced GL free energy becomes

$$
\Omega_{3 F}(\sigma, d)=\left(\frac{a}{2} \sigma^{2}-\frac{c}{3} \sigma^{3}+\frac{b}{4} \sigma^{4}+\frac{f}{6} \sigma^{6}\right)+\left(\frac{\alpha}{2} d^{2}+\frac{\beta}{4} d^{4}\right)-\gamma d^{2} \sigma+\lambda d^{2} \sigma^{2} .
$$

Some comments are in order on the coefficients of the terms in $\Omega_{3 F}(\sigma, d)$ :

(i) Changes in the magnitude of $a$ and $\alpha$ drive the phase transitions. In the following, we use these variables as the key parameters in classifying the phase structure.

(ii) As suggested in effective theories and in lattice QCD simulations [18], $b$ may change sign as a function of $T$ and $\mu$ (see Eq. (C13) in Appendix C). To incorporate such a possibility, we have introduced a $\mathcal{O}\left(\sigma^{6}\right)$ term with a coefficient $f>0$ to stabilize the system. As we will see later, the qualitative phase structure for three massless flavors is independent of the sign of $b$.

(iii) We assume that $\beta$ is always positive, as expected from effective theories and weak coupling QCD (see Eq. (C11) in Appeidix (C). This sign implies that the transition from the normal phase to CSC is second order at the tree-level if there is no coupling between $d$ and $\Phi$. Fluctuations of the diquark or gluon fields can make the transition first order [19], an effect we will not treat in this paper.

(iv) Because both the $d^{2} \sigma$ and $\sigma^{3}$ terms in Eq. (17) originate from the axial anomaly, their coefficients, $\gamma$ and $c$, are related microscopically. Indeed, it can be shown from the instanton-induced six-fermion interaction, $\sim \operatorname{det}_{i, j}\left(\bar{q}_{R}^{j} q_{L}^{i}\right)$, that $\gamma$ has the same sign and the same order of magnitude as $c$ (Appendix [B). Since $c$ is positive, as noted below Eq. (7), $\gamma$ is also positive. By lowering the free energy, the $\gamma$-term favors coexistence: $\sigma \neq 0$ and $d \neq 0$

(v) Microscopic calculations in weak coupling QCD and in the NJL model (Appendix C) show that the coefficient $\lambda$ is always positive. Physically a non-vanishing $\sigma$ plays the role of an effective mass for the quark field, reducing the density of states at the Fermi surface, and the pairing energy [15], an effect represented by $\lambda d^{2} \sigma^{2}>0$. Furthermore, as shown in Appendix $\mathrm{C}, \lambda / \beta \sim \ln \left(\Lambda / T_{d}\right) /\left(\mu / T_{d}\right)^{2}$, which is rather small for reasonable values of $\mu, T_{d}$ (the critical temperature of the color superconductivity without the $\sigma$ - $d$ coupling), and $\Lambda$ ( $\sim \mu$ for weak coupling QCD, and in the NJL model, of order the spatial momentum cutoff). Therefore, we focus primarily on the case $\gamma>0$ with $\lambda=0$ for the three-flavor case. The effect of the small positive $\lambda$ for three-flavor case is discussed in Appendix A.

In principle, the system can have four possible phases:

$$
\begin{array}{r}
\text { Normal (NOR) phase : } \sigma=0, d=0, \\
\text { CSC phase : } \sigma=0, d \neq 0, \\
\text { NG phase : } \sigma \neq 0, d=0, \\
\text { Coexistence (COE) phase : } \sigma \neq 0, d \neq 0 .
\end{array}
$$

The symmetry breaking pattern of the individual phases are the following: in the CSC phase, $\mathcal{G} \rightarrow S U(3)_{C+L+R} \times$ $Z(2)$; in the NG phase, $\mathcal{G} \rightarrow S U(3)_{C} \times S U(3)_{L+R} \times U(1)_{B}$, and in the COE phase, $\mathcal{G} \rightarrow S U(3)_{C+L+R} \times Z(2)$. Note that the CFL phase and COE phase with the diquark condensate of Eq. (16) each break the original $U(1)_{B} \times Z(6)_{A}$ symmetry with axial anomaly down to $Z(2)$ (the simultaneous reflection, $q_{L, R} \rightarrow-q_{L, R}$ ), and thus these two phases are not distinguished by symmetry.

\section{Outline of the phase diagram}

The phase diagram in the $a-\alpha$ plane can be determined uniquely by comparing the global minima of the free energies, $\Omega^{(\mathrm{NOR})}, \Omega^{(\mathrm{CSC})}, \Omega^{(\mathrm{NG})}$, and $\Omega^{(\mathrm{COE})}$. In the following, we outline the basic aspects of the phase diagram obtained from comparison of the free energies, and give the calculations of the phase boundaries and critical points in detail in Appendix A.

In the absence of the $\sigma$ - $d$ coupling $(\gamma=\lambda=0$ ), the four phases in (18) are separated by $\alpha=0$ (a line of second order transitions) and by $a=a_{\chi}$ (a line of first order phase transitions), shown in Fig 1 , where the first and second order phase boundaries are drawn as double and single lines, respectively. 


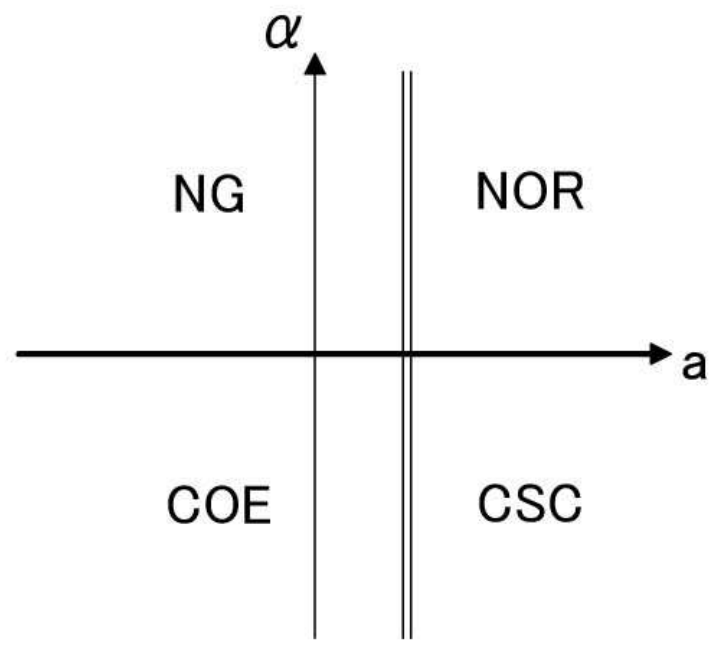

FIG. 1: Phase structure in three and two-flavor systems without coupling between the chiral and diquark condensates $(\gamma=$ $\lambda=0)$. Phase boundaries with a second order transition are denoted by a single line, and with a first order transition by a double line.

An attractive coupling, $\gamma>0$ (with $\lambda=0$ ) leads to several important modifications, summarized in (i)-(iv) below and shown in Fig 2 (the left panel for small $\gamma$ and the right panel for large $\gamma$ ). ${ }^{2}$

(i) The area of the COE phase grows when both $\sigma$ and $d^{2}$ are non-vanishing and positive, since the $-\gamma d^{2} \sigma$ term lowers the free energy.

(ii) The first order line between the COE and CSC phases, originally the double vertical line in Fig. 1, terminates at a critical point, A, as shown in Fig. 2. This behavior is anticipated, since $-\gamma d^{2}$ acts as an external field for $\sigma$ and washes out the first order phase transition for sufficiently large $\gamma$ or $d$. In Fig. 2 we denote the coexistence region contacting the NG phase as the NG-like COE phase, and the region which was originally the CSC phase as the CSC-like COE phase.

(iii) The second order phase boundary originally located at $\alpha=0$ splits in two, a line going to the right from the critical end point B and a line going to the left from the point C. Since $\sigma$ changes discontinuously across the first order phase boundary $\mathrm{CB}$, the $d^{2} \sigma$ term, which acts as a mass term for $d^{2}$, leads to different critical temperatures for diquark condensation in the NG-like COE and CSC-like COE phases.

(iv) For large enough $\gamma$, a tricritical point $\mathrm{D}$ appears on the boundary between the NG and COE phases. Then the point $\mathrm{C}$, otherwise a critical end point, becomes a triple point.

\section{Massless two-flavor case}

We turn now to the massless two-flavor system (with infinite strange quark mass). In this case, all chiral and diquark condensates with a strange quark are suppressed. We write

$$
\begin{aligned}
\Phi & =\operatorname{diag}(\sigma, \sigma, 0), \\
d_{L} & =-d_{R}=\operatorname{diag}(0,0, d) .
\end{aligned}
$$

\footnotetext{
${ }^{2}$ In describing the phase diagram we denote a point where a first order line turns into a crossover as a critical point; a point where a first order line turns into a second order line as a tricritical point; a point where a second order line terminates on a first order line as a critical end point; the intersection of three first order lines as a triple point; and a point where two or four second order lines meet as a bicritical or tetracritical point respectively [20].
} 

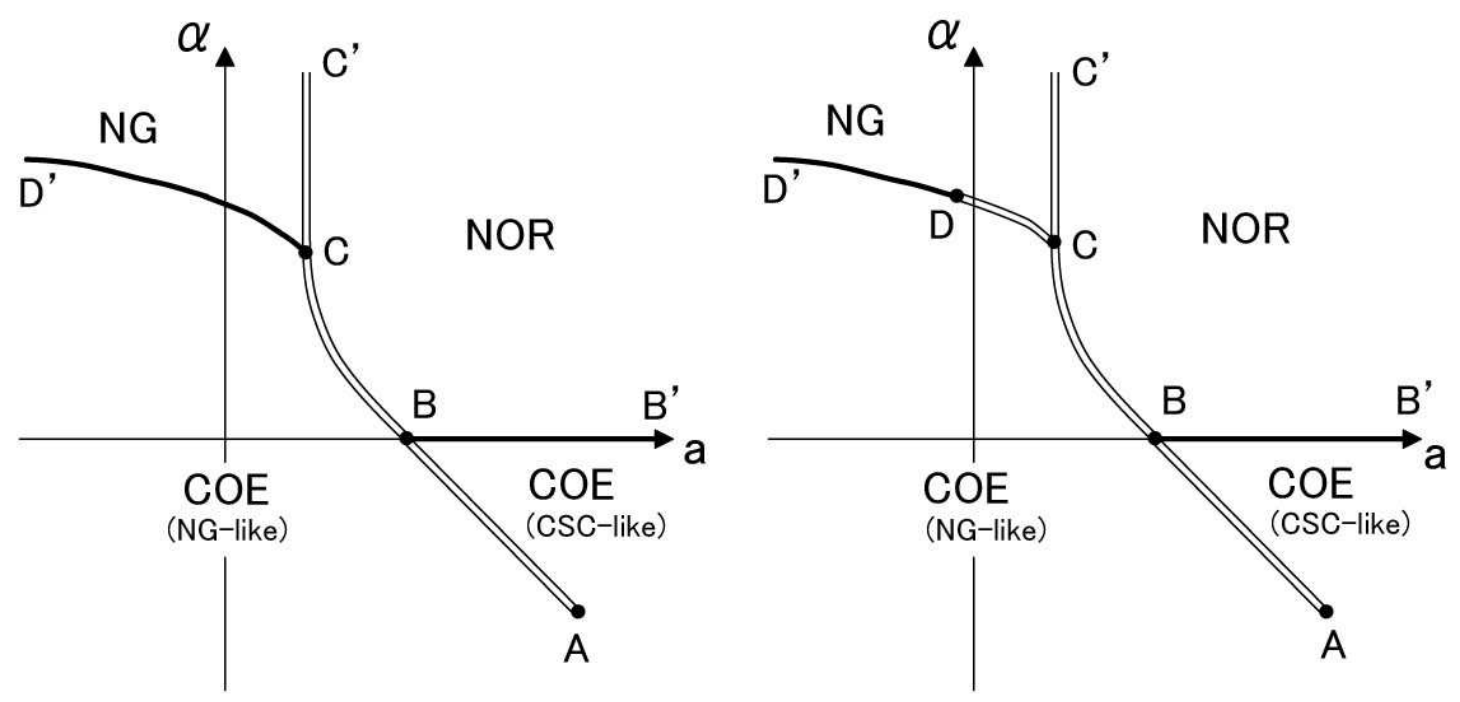

FIG. 2: Phase structure in the three-flavor system with $\gamma>0$ and $\lambda=0$. Phase boundaries with a second order transition are denoted by a single line, and with a first order transition by a double line. On the left, $\mathrm{C}$ is a critical end point $(0<\gamma<$ $(c / 3) \sqrt{\beta / b})$, and on the right, a triple point $(\gamma>(c / 3) \sqrt{\beta / b})$.

The latter is the two flavor color superconductivity (2SC) state. Due to this color-flavor structure, the cubic terms in $\sigma$ and $d$ are identically zero, and the model reduces to:

$$
\Omega_{2 F}(\sigma, d)=\left(\frac{a}{2} \sigma^{2}+\frac{b}{4} \sigma^{4}+\frac{f}{6} \sigma^{6}\right)+\left(\frac{\alpha}{2} d^{2}+\frac{\beta}{4} d^{4}\right)+\lambda d^{2} \sigma^{2} .
$$

We locate the phase boundaries and the order of the phase transitions by comparing the free energies: $\Omega^{(\mathrm{CSC})}(0, d)=$ $\frac{\alpha}{2} d^{2}+\frac{\beta}{4} d^{4}, \Omega^{(\mathrm{NG})}(\sigma, 0)=\frac{\alpha}{2} \sigma^{2}+\frac{b}{4} \sigma^{4}+\frac{f}{6} \sigma^{6}$ and $\Omega^{(\mathrm{COE})}(\sigma, d)$, with respect to $\Omega^{(\mathrm{NOR})}(0,0)$.

\section{Outline of the phase diagram}

We first consider $b>0$ in Eq. (21), with the $\sigma^{6}$ term safely neglected, to find the qualitative phase structure. The system is equivalent to an anisotropic antiferromagnet (e.g., $\mathrm{GdAlO}_{3}$ ) 20]. For $\lambda=0$, the boundaries of the four phases in (18) are characterized by second order lines at $\alpha=0$ and $a=0$ with a tetracritical point at $\alpha=a=0$. In the presence of the repulsive $d^{2} \sigma^{2}$ term $(\lambda>0)$, the area of the coexistence phase decreases, as shown in the left panel of Fig. 3. For $\lambda>\frac{1}{2} \sqrt{b \beta}$, the coexistence region disappears altogether, and a first order interface between CSC and NG appears at $\alpha=a \sqrt{\beta / b}$. In addition, $a=\alpha=0$ becomes a bicritical point, as shown in the right panel of Fig. 3.

Next we consider $b<0$; here the $\sigma^{6}$ term plays an essential role. For $\lambda=0$, the four phases in Eq. (18) are separated by a second order line at $\alpha=0$ and a first order line at $a=3 b^{2} /(16 f)$ as shown in Fig. 1 With a repulsive $d^{2} \sigma^{2}$ term $(\lambda>0)$ the coexistence phase shrinks and gradually fades away as $\lambda \rightarrow \infty$. Moreover, a new first order line between NG and CSC appears and grows as $\lambda$ increases. This situation is shown in Fig. 4 Such a phase structure was previously pointed out by Vanderheyden and Jackson 21] using the random matrix model, and by Kitazawa et al. 22] using the NJL model; our model-independent analysis is consistent with these results. However, the random matrix model does not include the physics of the suppression of the density of states at the Fermi surface leading to $\lambda>0$, and thus its predicted phase structure is not obviously related to that discussed here.

\section{Speculative phase structure for realistic quark masses}

The mapping of the phase diagrams obtained in the $(a, \alpha)$ plane to the $(T, \mu)$ plane is a dynamical question which we cannot address within the phenomenological GL theory. Nevertheless, on the basis of the results of Sec. II we can

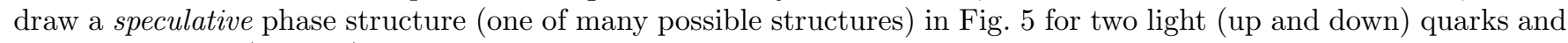
a medium-heavy (strange) quark. 

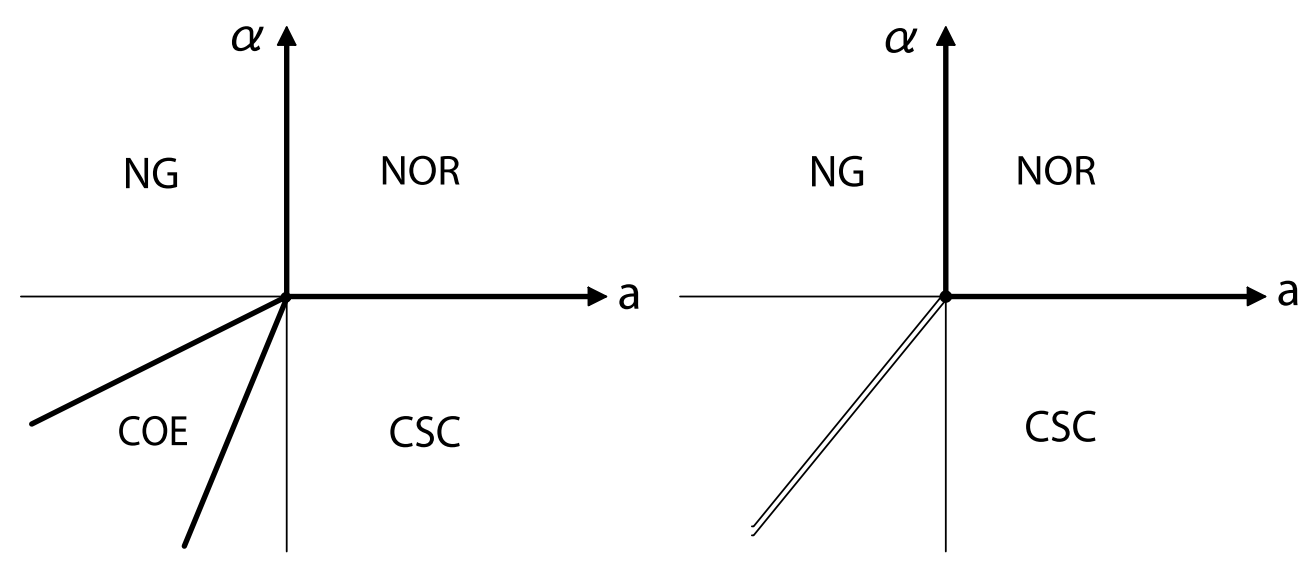

FIG. 3: Phase strucuture in the two-flavor system for $b>0$ and $\lambda>0$. Left: Case with a tetracritical point $\left(\frac{1}{2} \sqrt{b \beta}>\lambda>0\right)$. The second order line between NG and COE is characterized by $\alpha=2 a \lambda / b$, and that between CSC and COE by $\alpha=a \beta / 2 \lambda$. Right: Case with a bicritical point $\left(\lambda>\frac{1}{2} \sqrt{b \beta}\right)$. The first order line between NG and CSC is characterized by $\alpha=a \sqrt{\beta / b}$.

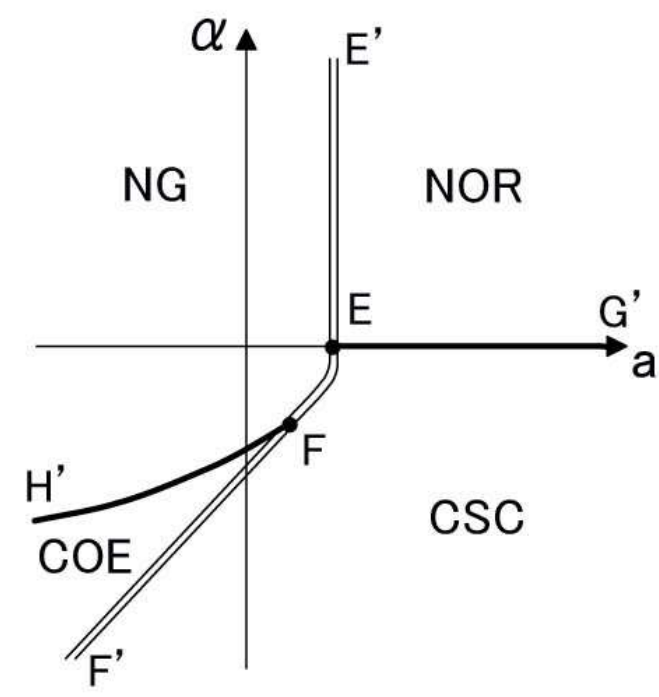

FIG. 4: Phase strucuture of the two-flavor system with $b<0$ and $\lambda>0$. The second order line between NG and COE is given by $\alpha=\lambda\left(b-\sqrt{b^{2}-4 f a}\right) / f$. The first order line between CSC and COE is given by $\alpha=(\beta / 2 \lambda)\left[a-3\left(b-4 \lambda^{2} / \beta\right)^{2} /(16 f)\right]$, while the first order line between NG and CSC is given by $\alpha^{2}=\beta\left[6 b f a+\left(b^{2}-4 f a\right)^{3 / 2}-b^{3}\right] /\left(6 f^{2}\right)$.

In the figure, we draw two critical points: the usual one near the vertical axis (the high temperature critical point) originally found by Asakawa and Yazaki [18], and a new one near the horizontal axis (the low temperature critical point) driven by the axial anomaly. We have assumed that both critical points are in the region of positive $T$ and $\mu$, and thus the chiral transition is a crossover in the direction of both high $T$ and high $\mu$. With decreasing strange quark mass, $m_{s}$, the high- $T$ critical point approaches the vertical axis because the quark mass, which tends to weaken the first order transiton, becomes less effective. On the other hand, as $m_{s}$ increases, the low- $T$ critical point approaches the horizontal axis since the system approaches the two-flavor case where the anomaly-induced critical point does not appear. Whether this scenario is realized or not needs to be checked in effective models incorporating the axial anomaly, and eventually be checked by first principles QCD simulations. Such calculations are beyond the scope of this paper.

We should mention a similar two-critical-point phase structure found in the two-flavor NJL model [22]. Since the axial anomaly does not produce a triple boson coupling in two flavors, as discussed in Sec. IIC the origin of the low- $T$ critical point in Ref. 22] is not related to ours, but can be understood as follows: Expanding the free energy of the two-flavor NJL model up to $\mathcal{O}\left(d^{2} \sigma^{4}\right)$. we arrive at an "effective" quartic term in the GL free energy,

$$
\frac{1}{4} b \sigma^{4}+\frac{1}{4} \tilde{b} d^{2} \sigma^{4}
$$


It can be shown that $b$ changes sign from positive to negative, and $\tilde{b}$ from negative to positive, as $\mu$ increases from zero. (See Eqs. (C13 and C14) in Appendix C]) Thus for sufficiently large $\mu$ and $d$, the first order transition driven by $b<0$ disappears due to $\tilde{b} d^{2}>0$. This implies that the critical point in [22] in the two-flavor case originates from a large higher order term in the GL expansion, and is thus beyond the reach of our strict GL expansion.

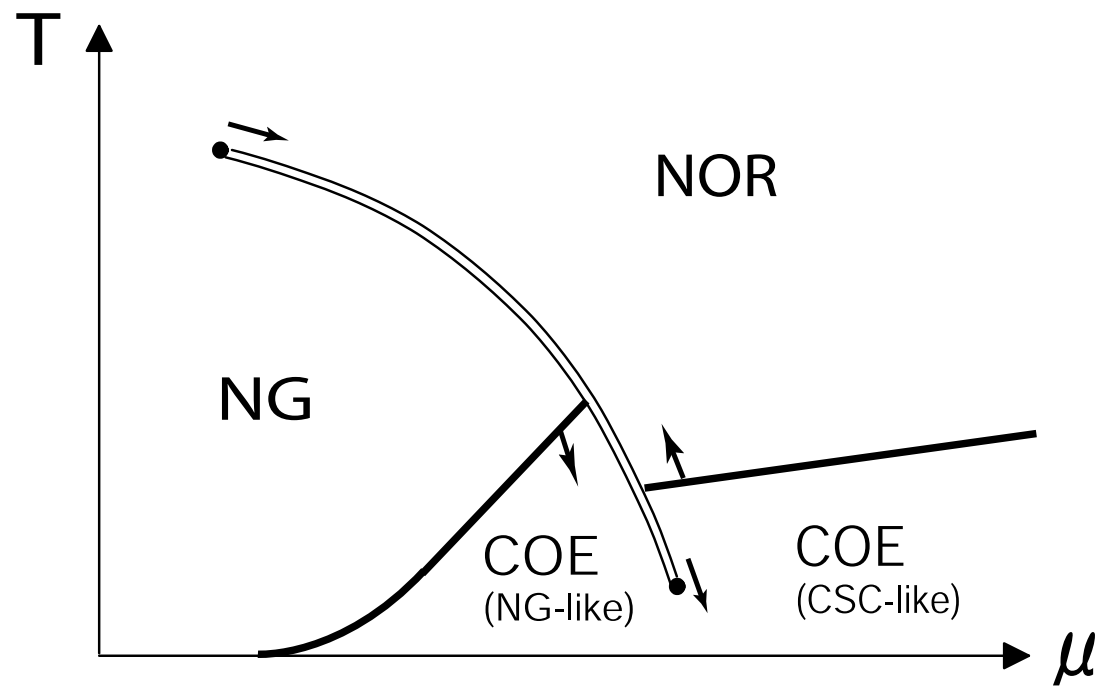

FIG. 5: Schematic phase structure with two light (up and down) quarks and a medium heavy (strange) quark. The arrows show how the critical point and the phase boundaries move as the strange-quark mass increases towards the two-flavor limit.

\section{EXCITATION SPECTRA OF MESONS}

We turn, in this section, to study the mass spectra of mesons including $H$ boson in the intermediate density region for the degenerate three-flavor case. (The $\eta^{\prime}$ is discussed in Appendix D, We focus on the region near the phase boundaries where $\sigma$ and $d$ are sufficiently small in the COE phase, and consider energy scales, $p$, smaller than the pairing gap $d$, so that we can neglect excitations of the quarks.

The symmetry breaking pattern for the chiral field at low density is $S U(3)_{L} \times S U(3)_{R} \rightarrow S U(3)_{V}$. Accordingly one has eight Nambu-Goldstone (NG) bosons, identified with the pseudoscalar $\bar{q} q$-state octet. These pions are massless in the chiral limit, with masses generated by finite quark masses. (The $\eta^{\prime}$ meson is much heavier than the pions because of the $U(1)_{A}$ axial anomaly.)

On the other hand, the pattern of symmetry breaking for the diquark fields in the asymptotic high density limit is $S U(3)_{C} \times S U(3)_{L} \times S U(3)_{R} \times U(1)_{B} \rightarrow S U(3)_{C+L+R} \times Z(2)_{B}$, leading altogether to $17 \mathrm{NG}$ bosons. Eight of these bosons are "eaten" by the gluons through the Anderson-Higgs mechanism; the remaining nine are the pseudoscalar octet $\tilde{\pi}$ (the "generalized pions," or $\bar{q} q q q$-states as discussed in [5, 16, 23, 24, 25]), together with the $H$ boson. $^{3}$

In the intermediate density region, two sets of light pseudoscalar mesons are present, one arising from the chiral condensate ( $\bar{q} q$-states) and the other from the diquark condensates ( $\bar{q} \bar{q} q q$-states); these two sets are mixed through the interaction terms [23]. Specifically, we have two sets of pion octets $\left(\pi\right.$ and $\tilde{\pi}$ ) (as well as $\eta^{\prime}$ and $\left.\tilde{\eta}^{\prime}\right)$ from the chiral and diquark fields, and in addition the $H$ boson from the diquark field in this region. As we show below, mixing causes one of the two pions to become heavy while the other becomes massless. (A similar discussion of the mixing between $\bar{q} q$ - and $\bar{q} \bar{q} q q$-states at $T=0$ and $\mu=0$ is given in [26].) The $H$ boson does not experience mixing, since it exists only in the diquark sector, but remains exactly massless independent of the quark masses.

In the following, we examine the mass spectra corresponding to this physical picture more quantitatively using an effective Lagrangian approach. We begin with a toy model with independent massless fields to show explicitly the essential features of the role mixing plays. Next we construct an effective Lagrangian involving the pion octet appropriate in the intermediate density region. Using the nonlinear chiral Lagrangians studied for the NG phase

\footnotetext{
${ }^{3}$ In addition, one also has a massive $\tilde{\eta}^{\prime}$, since $U(1)_{A}$ remains broken by the axial anomaly in the high density limit. However, the violation of $U(1)_{A}$ symmetry becomes much smaller as screening suppresses the instanton density by a high power of $1 / \mu$ [17]. Correspondingly we have a light $\eta^{\prime}$ meson at asymptotically high density.
} 
[27] and for the CFL phase [16, 24, 28, 29], we take into account $\pi$ and $\tilde{\pi}$ and study the effects of the mixing terms determined in Sec. III, which were previously not considered. The diagonalization of the mixing matrix for $\pi$ and $\tilde{\pi}$ leads to a generalized Gell-Mann-Oakes-Renner (GOR) relation in dense QCD, which relates the masses of pseudoscalar bosons to the magnitude of the chiral and diquark condensates. We also discuss the continuity between the NG and the CSC phases associated with the excited states.

\section{A. A toy model}

To show the effect the mixing of independent fields in the presence of mass terms, we first consider a simple toy model for two complex scalar fields, $\alpha$ and $\tilde{\alpha}$ :

$$
\begin{aligned}
\mathcal{L} & =\mathcal{L}_{\text {kin }}+\mathcal{L}_{\text {mix }}+\mathcal{L}_{\text {mass }} \\
\mathcal{L}_{\text {kin }} & =\frac{f^{2}}{2}\left|\partial_{\mu} \alpha\right|^{2}+\frac{\tilde{f}^{2}}{2}\left|\partial_{\mu} \tilde{\alpha}\right|^{2}, \\
\mathcal{L}_{\text {mix }} & =\frac{A}{2}\left(\alpha \tilde{\alpha}^{\dagger}+\text { h.c. }\right) \\
\mathcal{L}_{\text {mass }} & =\frac{m}{2}(B \alpha+C \tilde{\alpha}+\text { h.c. }),
\end{aligned}
$$

where $A, B, C, f, \tilde{f}$ and $m$ are positive constants.

We focus on the low mass phase excitations, described by the fields $\varphi$ and $\tilde{\varphi}$ defined by

$$
\alpha=\exp (i \varphi / f), \quad \tilde{\alpha}=\exp (i \tilde{\varphi} / \tilde{f}) .
$$

In the absence of the mixing term $\mathcal{L}_{\text {mix }}$ and mass term $\mathcal{L}_{\text {mass }}$, both $\varphi$ and $\tilde{\varphi}$ are massless. We turn on $\mathcal{L}_{\text {mix }}$ and $\mathcal{L}_{\text {mass }}$, and expand the Lagrangian in terms of $\varphi$ and $\tilde{\varphi}$. After subtracting a constant part, we obtain

$$
\begin{aligned}
\mathcal{L}_{\text {mix }}+\mathcal{L}_{\text {mass }} & =-\frac{1}{2}\left(\begin{array}{ll}
\varphi & \tilde{\varphi}
\end{array}\right) M_{(\varphi)}\left(\begin{array}{c}
\varphi \\
\tilde{\varphi}
\end{array}\right)+\mathcal{O}\left(\varphi^{4}\right), \\
M_{(\varphi)} & =\left(\begin{array}{cc}
(A+m B) / f^{2} & -A / f \tilde{f} \\
-A / f \tilde{f} & (A+m C) / \tilde{f}^{2}
\end{array}\right) .
\end{aligned}
$$

The matrix $M_{(\varphi)}$ is diagonalized by a transformation

$$
\left(\begin{array}{l}
\varphi_{1} \\
\varphi_{2}
\end{array}\right)=\left(\begin{array}{cc}
\cos \Theta & \sin \Theta \\
-\sin \Theta & \cos \Theta
\end{array}\right)\left(\begin{array}{c}
\varphi \\
\tilde{\varphi}
\end{array}\right)
$$

where $\Theta$ is the mixing angle; we thus we obtain the eigenmasses $m_{\varphi_{1}}^{2}$ and $m_{\varphi_{2}}^{2}\left(m_{\varphi_{1}}^{2}<m_{\varphi_{2}}^{2}\right)$ :

(i) For $m=0$, we have

$$
\begin{aligned}
& m_{\varphi_{1}}^{2}(m=0)=0 \\
& m_{\varphi_{2}}^{2}(m=0)=A\left(\frac{1}{f^{2}}+\frac{1}{\tilde{f}^{2}}\right) .
\end{aligned}
$$

The presence of a massless excitation corresponds to simultaneous rotation of $\varphi_{1}$ and $\varphi_{2}$ in the same direction, while the massive mode corresponds to rotation in opposite directions.

(ii) For small but non-vanishing $m$, we obtain

$$
\begin{aligned}
& m_{\varphi_{1}}^{2} \simeq \frac{m}{f^{2}+\tilde{f}^{2}}(B+C), \\
& m_{\varphi_{2}}^{2} \simeq m_{\varphi_{2}}^{2}(m=0)+\frac{m}{f^{2}+\tilde{f}^{2}}\left(B \frac{\tilde{f}^{2}}{f^{2}}+C \frac{f^{2}}{\tilde{f}^{2}}\right),
\end{aligned}
$$

In Sec. III we will discuss mass relations of this kind arising via mixing in the realistic situation of QCD. In particular, Eq. (29) is a key relation corresponding to our later generalized Gell-Mann-Oakes-Renner relation, Eq. (56). 


\section{B. General effective Lagrangian}

To derive the effective Lagrangian for the light excited states, the pions $(\pi, K$ and $\eta), \eta^{\prime}$ and the $H$ boson in the intermediate density region, we fix, as above, the magnitude of the chiral and diquark fields and consider only fluctuations of their phases about their vacuum configurations. We thus parametrize the fields as

$$
\Phi=\sigma \Sigma e^{-2 i \theta}, \quad d_{L}=d U_{L} e^{2 i(\tilde{\theta}+\phi)}, \quad d_{R}=-d U_{R} e^{-2 i(\tilde{\theta}-\phi)},
$$

where $\Sigma, U_{L}$ and $U_{R}$ are $S U(3)$ matrices, and the angles $\theta$ and $\tilde{\theta}$ are $U(1)_{A}$ phases, and $\phi$ the $U(1)_{B}$ phase.

As mentioned at the top of Sec. III all eight gluons acquire a mass of order $g f_{\tilde{\pi}} \sim \mathcal{O}(g \mu)$ by "eating" the eight colored fluctuations of $U_{L, R}$, where $g$ is the QCD coupling constant and $f_{\tilde{\pi}}$ is the decay constant associated with $U_{L, R}$, defined below. On the low momentum scales we consider, $p<d \ll g f_{\tilde{\pi}}$, gluons are not low-lying modes. The remaining color-singlet fluctuations correspond to $\tilde{\pi}$ and are parametrized by the field

$$
\tilde{\Delta}=U_{L} U_{R}^{\dagger}
$$

which transforms under $S U(3)_{L} \times S U(3)_{R}$ as

$$
\tilde{\Delta} \rightarrow V_{L} \tilde{\Delta} V_{R}^{\dagger}
$$

\section{Kinetic terms}

The kinetic term invariant under $\mathcal{G}$ for the chiral fields $\Sigma$ and $\theta$, to second order in derivatives is,

$$
\mathcal{L}_{\chi}^{\mathrm{kin}}=f_{\pi}^{2} g_{\pi}^{\mu \nu} \operatorname{Tr}\left(\partial_{\mu} \Sigma \partial_{\nu} \Sigma^{\dagger}\right)+\frac{f_{\eta^{\prime}}^{2}}{2} g_{\eta^{\prime}}^{\mu \nu} \partial_{\mu} \theta \partial_{\nu} \theta
$$

where the metric tensors $g_{\pi}^{\mu \nu}=\operatorname{diag}\left(1, v_{\pi}^{2}, v_{\pi}^{2}, v_{\pi}^{2}\right)$ and $g_{\eta^{\prime}}^{\mu \nu}=\operatorname{diag}\left(1, v_{\eta^{\prime}}^{2}, v_{\eta^{\prime}}^{2}, v_{\eta^{\prime}}^{2}\right)$ arise due to the absence of Lorentz invariance in the medium; Here $v_{\pi}$ and $v_{\eta^{\prime}}$ are the speeds of the pions and $\eta^{\prime}$, respectively.

The standard pion fields $\pi^{j}$ are defined by

$$
\Sigma=\exp \left(i \lambda^{j} \pi^{j} / f_{\pi}\right)
$$

where the $\lambda^{j}(j=1, \ldots, 8)$ are Gell-Mann matrices normalized so that $\operatorname{Tr} \lambda^{i} \lambda^{j}=\frac{1}{2} \delta^{i j}$. The first term in Eq. (34) is the standard leading-order chiral Lagrangian [27], except that the speed of the mesons in the medium differs from the speed of light and the Lagrangian contains additional contribution from the field $\theta$.

Following the discussions of Refs. [16] and [24], the most general Lagrangian of the diquark kinetic term invariant under $\mathcal{G}$ with two derivatives is

$$
\mathcal{L}_{d}^{\mathrm{kin}}=f_{\tilde{\pi}}^{2} g_{\tilde{\pi}}^{\mu \nu} \operatorname{Tr}\left(\partial_{\mu} \tilde{\Delta} \partial_{\nu} \tilde{\Delta}^{\dagger}\right)+\frac{f_{\tilde{\eta}^{\prime}}^{2}}{2} g_{\tilde{\eta}^{\prime}}^{\mu \nu} \partial_{\mu} \tilde{\theta} \partial_{\nu} \tilde{\theta}+\frac{f_{H}^{2}}{2} g_{H}^{\mu \nu} \partial_{\mu} \phi \partial_{\nu} \phi,
$$

with the additional metric tensor $g_{H}^{\mu \nu}=\operatorname{diag}\left(1, v_{H}^{2}, v_{H}^{2}, v_{H}^{2}\right)$. The generalized pion fields $\tilde{\pi}^{j}$ are defined by

$$
\tilde{\Delta}=\exp \left(i \lambda^{j} \tilde{\pi}^{j} / f_{\tilde{\pi}}\right) .
$$

The three terms in Eq. (36) are the kinetic terms for pions, $\eta^{\prime}$, and the $H$ boson, respectively [16, 24, 28].

At extremely high density, the decay constants $f_{\tilde{\pi}}, f_{\tilde{\eta}^{\prime}}$, and $f_{H}$ are found by matching to their microscopic asymptotic values [16]:

$$
f_{\tilde{\pi}}^{2} \rightarrow \frac{21-8 \ln 2}{18} \frac{\mu^{2}}{2 \pi^{2}}, \quad f_{H, \tilde{\eta^{\prime}}}^{2} \rightarrow \frac{3}{4} \frac{\mu^{2}}{2 \pi^{2}},
$$

while the velocities approach the asymptotic sound speed: $v_{\tilde{\pi}, \tilde{\eta}^{\prime}, H}^{2} \rightarrow 1 / 3$. 


\section{Mass terms}

We now assume small but finite quark masses and construct the possible terms in the GL free-energy allowed by the symmetry group $\mathcal{G}$, where $M$ is the $3 \times 3$ quark mass matrix in flavor space. In the intermediate density region, where $U(1)_{A}$ symmetry is violated by the axial anomaly, the lowest order possible mass terms in the GL free-energy are $\mathcal{O}(M)$, unlike at asymptotically high density where the leading terms are $\mathcal{O}\left(M^{2}\right)$.

The bare quark mass term in QCD reads

$$
\mathcal{L}_{\mathrm{QCD}}^{\operatorname{mass}}=\bar{q}_{L} M q_{R}+\text { h.c.. }
$$

As in the standard procedure to build up the chiral Lagrangian, we treat the matrix $M$ as a spurion field and assume it to transform under $\mathcal{G}$ as,

$$
M \rightarrow e^{-2 i \alpha_{A}} V_{L} M V_{R}^{\dagger}
$$

This transformation law together with Eqs. (5), (10) and (11) enable us to write down the possible mass terms. It should be stressed that $\Phi, d_{L} d_{R}^{\dagger}$ and $M$ share the same transformation property under $\mathcal{G}$ except for the $U(1)_{A}$ phase rotation. Therefore, the guiding principle to construct the quark mass terms is to replace the $\Phi$ 's or $d_{L} d_{R}^{\dagger}$ 's involved in the GL potential $\Omega_{\chi, d, \chi d}$, in Eqs. (7), (13) and (14), with $M$.

The general mass terms of the chiral and diquark fields obtained from this procedure are:

$$
\begin{aligned}
\mathcal{L}^{\text {mass }}= & A_{0}\left[\operatorname{Tr}\left(M \Phi^{\dagger}\right)+\text { h.c. }\right] \\
& +B_{0}\left[\varepsilon_{a b c} \varepsilon_{i j k} M_{a i} \Phi_{b j} \Phi_{c k}+\text { h.c. }\right] \\
& +C_{1}\left[\operatorname{Tr}\left(M \Phi^{\dagger}\right) \operatorname{Tr}\left(\Phi \Phi^{\dagger}\right)+\text { h.c. }\right] \\
& +C_{2}\left[\operatorname{Tr}\left\{\left(M \Phi^{\dagger}\right)\left(\Phi \Phi^{\dagger}\right)\right\}+\text { h.c. }\right] \\
& +\Gamma_{1}\left[\operatorname{Tr}\left\{M\left(d_{R} d_{L}^{\dagger}\right)\right\}+\text { h.c. }\right] \\
& +\Lambda_{1}\left[\operatorname{Tr}\left\{\left(d_{L} d_{L}^{\dagger}+d_{R} d_{R}^{\dagger}\right)\left(M \Phi^{\dagger}\right)\right\}+\text { h.c. }\right] \\
& +\Lambda_{2}\left[\operatorname{Tr}\left(d_{L} d_{L}^{\dagger}+d_{R} d_{R}^{\dagger}\right) \operatorname{Tr}\left(M \Phi^{\dagger}\right)+\text { h.c. }\right] \\
& +\Lambda_{3}\left[\varepsilon_{a b c} \varepsilon_{i j k} M_{a i} \Phi_{b j}\left(d_{L} d_{R}^{\dagger}\right)_{c k}+\text { h.c. }\right] .
\end{aligned}
$$

Note that the terms proportional to $B_{0}$ and $\Gamma_{1}$, which break $U(1)_{A}$ symmetry, originate from the axial anomaly, or equivalently, by instanton-induced interactions. The leading mass terms, which are linear in $M$ and have minimal number of the chiral and diquark fields, become

$$
\mathcal{L}^{\text {mass }}=A_{0}\left[\operatorname{Tr}\left(M \Phi^{\dagger}\right)+\text { h.c. }\right]+\Gamma_{1}\left[\operatorname{Tr}\left\{M\left(d_{R} d_{L}^{\dagger}\right)\right\}+\text { h.c. }\right] \text {. }
$$

\section{Overall effective Lagrangian}

We turn now to the overall effective Lagrangian for $\pi, \eta^{\prime}$ and $H$. With the parametrizations of the fields defined in Eqs. (31) and (32), the GL potential of the chiral part $\Omega_{\chi}$ in Eq. (7) and the interaction part $\Omega_{\chi d}$ in Eq. (14) reduce to

$$
\begin{aligned}
\mathcal{L}_{\chi} & =c_{0} \sigma^{3} \cos (6 \theta)+\text { const. } \\
\mathcal{L}_{\chi d} & =\gamma_{1} d^{2} \sigma\left[e^{-2 i(2 \tilde{\theta}+\theta)} \operatorname{Tr}\left(\tilde{\Delta}^{\dagger} \Sigma\right)+\text { h.c. }\right]+\lambda_{3} d^{2} \sigma^{2}\left[e^{2 i(\tilde{\theta}-\theta)} \operatorname{Tr}\left(\tilde{\Delta}^{\dagger} \Sigma\right)+\text { h.c. }\right]+\text { const. },
\end{aligned}
$$

while the diquark part $\Omega_{d}$ in Eq. (13) reduces to a constant and can be neglected. From Eqs. (34), (36), (42), (43) and (44), the overall effective Lagrangian takes the form up to $\mathcal{O}\left(\pi^{2}, \tilde{\pi}^{2}, \eta^{\prime 2}, \tilde{\eta}^{\prime 2}\right)$ :

$$
\begin{aligned}
\mathcal{L}^{\mathrm{eff}} & =\mathcal{L}_{(\pi)}^{\mathrm{eff}}+\mathcal{L}_{(H)}^{\mathrm{eff}}+\mathcal{L}_{\left(\eta^{\prime}\right)}^{\mathrm{eff}}, \\
\mathcal{L}_{(\pi)}^{\mathrm{eff}} & =\frac{1}{2} g_{\pi}^{\mu \nu}\left(\partial_{\mu} \pi^{j}\right)\left(\partial_{\nu} \pi^{j}\right)^{\dagger}+\frac{1}{2} g_{\tilde{\pi}}^{\mu \nu}\left(\partial_{\mu} \tilde{\pi}^{j}\right)\left(\partial_{\nu} \tilde{\pi}^{j}\right)^{\dagger}-\frac{1}{2}(\pi \quad \tilde{\pi}) M_{(\pi)}\left(\begin{array}{c}
\pi \\
\tilde{\pi}
\end{array}\right), \\
\mathcal{L}_{(H)}^{\mathrm{eff}} & =\frac{1}{2} g_{H}^{\mu \nu}\left(\partial_{\mu} H\right)\left(\partial_{\nu} H\right)^{\dagger}, \\
\mathcal{L}_{\left(\eta^{\prime}\right)}^{\mathrm{eff}} & =\frac{1}{2} g_{\eta^{\prime}}^{\mu \nu}\left(\partial_{\mu} \eta^{\prime}\right)\left(\partial_{\nu} \eta^{\prime}\right)^{\dagger}+\frac{1}{2} g_{\tilde{\eta}^{\prime}}^{\mu \nu}\left(\partial_{\mu} \tilde{\eta}^{\prime}\right)\left(\partial_{\nu} \tilde{\eta}^{\prime}\right)^{\dagger}-\frac{1}{2}\left(\begin{array}{ll}
\eta^{\prime} & \tilde{\eta}^{\prime}
\end{array}\right) M_{\left(\eta^{\prime}\right)}\left(\begin{array}{c}
\eta \\
\tilde{\eta}^{\prime}
\end{array}\right),
\end{aligned}
$$




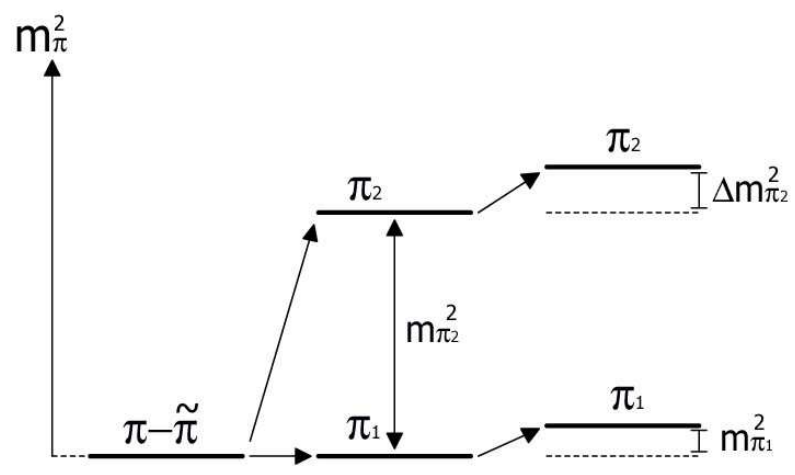

(a)

(b)

(c)

FIG. 6: Mass spectra of pions in the intermediate density region: (a) without $\sigma$ - $d$ mixing $(\gamma=\lambda=0$ ); (b) with $\sigma$ - $d$ mixing $(\gamma \neq 0, \lambda \neq 0)$; and $(\mathrm{c})$ in addition with finite quark masses $\left(m_{q} \neq 0\right)$. Explicit expressions for the $m_{\pi}^{2}$ 's are given in the text.

where the quark mass matrix is assumed to be diagonal and flavor symmetric, $M=\operatorname{diag}\left(m_{q}, m_{q}, m_{q}\right)$. The fields are redefined as

$$
\theta=\frac{\eta^{\prime}}{f_{\eta^{\prime}}}, \quad \tilde{\theta}=\frac{\tilde{\eta}^{\prime}}{f_{\tilde{\eta}^{\prime}}}, \quad \phi=\frac{H}{f_{H}} .
$$

The mass matrices of $(\pi, \tilde{\pi})$ and $\left(\eta^{\prime}, \tilde{\eta}^{\prime}\right)$ are

$$
\begin{aligned}
& M_{(\pi)}=\left(\begin{array}{cc}
\frac{1}{f_{\pi}^{2}}\left(\gamma_{1} d^{2} \sigma+\lambda_{3} d^{2} \sigma^{2}+A_{0} m_{q} \sigma\right) & -\frac{1}{f_{\pi} f_{\tilde{\pi}}}\left(\gamma_{1} d^{2} \sigma+\lambda_{3} d^{2} \sigma^{2}\right) \\
-\frac{1}{f_{\pi} f_{\tilde{\pi}}}\left(\gamma_{1} d^{2} \sigma+\lambda_{3} d^{2} \sigma^{2}\right) & \frac{1}{f_{\tilde{\pi}}^{2}}\left(\gamma_{1} d^{2} \sigma+\lambda_{3} d^{2} \sigma^{2}+\Gamma_{1} m_{q} d^{2}\right)
\end{array}\right) . \\
& M_{\left(\eta^{\prime}\right)}=12\left(\begin{array}{cc}
\frac{1}{f_{\eta^{\prime}}^{2}}\left(3 c_{0} \sigma^{3}+2 \gamma_{1} d^{2} \sigma+2 \lambda_{3} d^{2} \sigma^{2}+2 A_{0} m_{q} \sigma\right) & \frac{1}{f_{\eta^{\prime}} f_{\tilde{\eta}^{\prime}}}\left(4 \gamma_{1} d^{2} \sigma-2 \lambda_{3} d^{2} \sigma^{2}\right) \\
\frac{1}{f_{\eta^{\prime}} f_{\tilde{\eta}^{\prime}}}\left(4 \gamma_{1} d^{2} \sigma-2 \lambda_{3} d^{2} \sigma^{2}\right) & \frac{1}{f_{\tilde{\eta}^{\prime}}^{2}}\left(8 \gamma_{1} d^{2} \sigma+2 \lambda_{3} d^{2} \sigma^{2}+8 \Gamma_{1} m_{q} d^{2}\right)
\end{array}\right) .
\end{aligned}
$$

Since we have degenerate masses of up, down, and strange quarks, the Bedaque-Schäfer term [30] originating from the effective modification of the chemical potential due to the mass differences does not appear here. The off-diagonal components of $M_{(\pi)}$ originate from the chiral-diquark coupling. As the form of $M_{(\pi)}$ shows, both $\pi$ and $\tilde{\pi}$ are exactly massless in the absence of $\sigma-d$ coupling and quark masses.

\section{Mass spectra of low-lying collective modes}

In this subsection, we investigate the mass spectra of pions and $H$ on the basis of the effective Lagrangian obtained in the last section. (For $\eta^{\prime}$, see Appendix D, In particular, we obtain a generalized Gell-Mann-Oakes-Renner (GOR) relation in the intermediate density region. We also discuss a possible connection to hadron-quark continuity for these excited states.

\section{H boson}

As we see from Eq. (47), the $H$ boson is exactly massless irrespective of the quark masses. This is because the $H$ boson only exists in the diquark part and is not affected by the chiral-diquark coupling, so that there are no mass terms for $H$ involving the quark masses. On the other hand, the mass spectra of $\pi$ and $\eta^{\prime}$, unlike the $H$ boson, are non-trivial on account of mixing through the chiral-diquark coupling.

\section{Pions}

The eigenstates of the mass matrix $M_{(\pi)}$ in Eq. (50) can be written as

$$
\left(\begin{array}{l}
\pi_{1} \\
\pi_{2}
\end{array}\right)=\left(\begin{array}{cc}
\cos \vartheta & \sin \vartheta \\
-\sin \vartheta & \cos \vartheta
\end{array}\right)\left(\begin{array}{l}
\pi \\
\tilde{\pi}
\end{array}\right)
$$


with mixing angle $\vartheta$. We compute the pion masses $m_{\pi_{1}}$ and $m_{\pi_{2}}$ (we take $m_{\pi_{1}}^{2}<m_{\pi_{2}}^{2}$ ) with and without quark masses.

(i) In the chiral limit, $m_{q}=0$, the pion masses are

$$
\begin{aligned}
& m_{\pi_{1}}^{2}\left(m_{q}=0\right)=0 \\
& m_{\pi_{2}}^{2}\left(m_{q}=0\right)=\left(\gamma_{1} d^{2} \sigma+\lambda_{3} d^{2} \sigma^{2}\right) \frac{f_{\pi}^{2}+f_{\tilde{\pi}}^{2}}{f_{\pi}^{2} f_{\tilde{\pi}}^{2}}
\end{aligned}
$$

with the mixing angle $\vartheta_{0}$, which satisfies

$$
\tan \vartheta_{0}=\frac{f_{\tilde{\pi}}}{f_{\pi}}
$$

(ii) For non-vanishing $m_{q}$, we find

$$
\begin{aligned}
& m_{\pi_{1}}^{2}=\frac{m_{q}}{f_{\pi}^{2}+f_{\tilde{\pi}}^{2}}\left(A_{0} \sigma+\Gamma_{1} d^{2}\right) \\
& m_{\pi_{2}}^{2}=m_{\pi_{2}}^{2}\left(m_{q}=0\right)+\frac{m_{q}}{f_{\pi}^{2}+f_{\tilde{\pi}}^{2}}\left(\frac{f_{\tilde{\pi}}^{2}}{f_{\pi}^{2}} A_{0} \sigma+\frac{f_{\pi}^{2}}{f_{\tilde{\pi}}^{2}} \Gamma_{1} d^{2}\right),
\end{aligned}
$$

with the mixing angle

$$
\tan \vartheta=\frac{f_{\tilde{\pi}}}{f_{\pi}}+\frac{f_{\pi}}{f_{\tilde{\pi}}} \frac{f_{\pi}^{2} \Gamma_{1} d^{2}-f_{\tilde{\pi}}^{2} A_{0} \sigma}{\left(f_{\pi}^{2}+f_{\tilde{\pi}}^{2}\right)\left(\gamma_{1} d^{2} \sigma+\lambda_{3} d^{2} \sigma^{2}\right)} m_{q} .
$$

The resultant mass spectra of pions is shown in Fig. 6. Panel (a) shows the case with neither the chiral-diquark coupling nor finite quark masses. In this case both $\pi$ and $\tilde{\pi}$ are massless and degenerate. Figure 6 (b) shows the effects of the chiral-diquark coupling without finite quark masses; here the masses are split, and a massless mode still remains. Panel (c) shows the effects of both chiral-diquark coupling and finite quark masses; here because of the explicit breaking of chiral symmetry by quark masses, the masses of $\pi_{1}$ and $\pi_{2}$ are lifted by $\Delta m_{\pi_{1}}^{2}$ and $\Delta m_{\pi_{2}}^{2}$ respectively. The mass formula for the lightest pseudoscalar meson, Eq. (56), is a generalized form in dense QCD of the Gell-Mann-Oakes-Renner (GOR) relation connecting the masses of pseudoscalar bosons to the chiral and diquark condensates. The $\Gamma_{1}$ term, which originates from the effect of the axial anomaly in the pion Lagrangian, $\mathcal{L}_{(\pi)}^{\text {eff }}$, shows the crucial role of the axial anomaly not only for the phase structure but also for the excitation spectra in the intermediate density region. When the diquark condensate $d$ decreases as the density becomes low, Eq. (566) reduces to the standard GOR relation $f_{\pi}^{2} m_{\pi}^{2}=A_{0} m_{q} \sigma$. On the other hand, at asymptotically high density, the chiral condensate $\sigma$ is small and the axial anomaly is highly suppressed as $\Gamma_{1} \sim \mu\left(\Lambda_{\mathrm{QCD}} / \mu\right)^{9}(1 / g)^{14}[17]$. Then, the linear term in $m_{q}$ disappears on the right side of Eq. (56) and the leading term becomes $\mathcal{O}\left(m_{q}^{2}\right)$, with $m_{\pi_{1}}^{2} \propto m_{q}^{2} d^{2}$. This result is consistent with observations given in [16, 31] at high density.

Note that $\pi_{2}$ is much heavier than $\pi_{1}$ and it is thus expected to be radically unstable against decay into $\pi_{1}$. The surviving excitation mode $\pi_{1}$ exhibits hadron-quark continuity with increasing baryon density. The mixing angle $\vartheta=0$ describes the low density limit $\left(\pi_{1}=\pi\right)$ and $\vartheta=\pi / 2$ the asymptotically high density limit $\left(\pi_{1}=\tilde{\pi}\right)$. In the intermediate density region, $\pi_{1}$ is the result of mixing between the $\sigma \sim \bar{q} q$ state, as seen in the low density region, and the $d^{2} \sim \bar{q} \bar{q} q q$ state, as seen in the high density region. The mixing angle $\vartheta$ changes with the chemical potential $\mu$. Therefore, across the entire span of the chemical potential, we have light pseudoscalar modes associated with chiral symmetry breaking, indicating hadron-quark continuity for the excited pionic states.

\section{SUMMARY AND CONCLUSION}

In this paper, we have investigated the phase structure and excited spectra of dense QCD in the presence of the axial anomaly. We constructed the general GL free-energy and effective Lagrangian for the chiral, diquark fields, and their interactions, consistent with QCD symmetry, $\mathcal{G} \equiv S U\left(N_{f}\right)_{L} \times S U\left(N_{f}\right)_{R} \times U(1)_{B} \times U(1)_{A} \times S U(3)_{C}$.

In Sec. III we have shown that the QCD axial anomaly acts as an external field for the chiral condensate under the influence of the diquark condensate. The first order chiral transition is changed to crossover for a large diquark condensate, and a new critical point driven by the axial anomaly emerges in the QCD phase diagram. The former is a realization of hadron-quark continuity for the QCD ground states. Determining the precise location of this new critical 
point is a future task for phenomenological models and lattice QCD simulations. Our schematic phase diagram would be made more realistic by including effects such as finite quark masses, charge neutrality, $\beta$-equilibrium, and thermal gluon fluctuations [15, 19]. Open questions include whether the new critical point would survive in an inhomogeneous Fulde-Ferrell-Larkin-Ovchinnikov (FFLO) state, and how the COE phase at low $T$ and low $\mu$, Fig. 5 , is affected by quark confinement.

In Sec. III, we have shown that the massless pions in the chiral limit in dense QCD result from the mixing of $\pi \sim q \bar{q}$ and $\tilde{\pi} \sim q q \bar{q} \bar{q}$ states. Turning on a small but finite quark mass, we found that the pions acquire a mass both from the chiral condensate $\sigma$ and the diquark condensate $d^{2}$, which is summarized in the generalized Gell-Mann-Oakes-Renner formula, Eq. (56). Here the QCD axial anomaly plays a key role, giving the $d^{2}$-contribution to the pion mass. Our results show explicitly that hadron-quark continuity is realized not only in the ground state but also in the low-lying collective excitations. The continuity of the heavier excitations such as the vector mesons and baryons remains an interesting problem for future exploration.

In Sec. III, we focused on the COE phase with three degenerate flavors. However, in the intermediate density region, in which we are interested, flavor symmetry breaking due to $m_{\mathrm{s}} \gg m_{\mathrm{u}, \mathrm{d}}$ is non-negligible and causes mass splittings of the octet of pions in a non-trivial way. In particular, it would be interesting to study how, across the intermediate density region, the inverse mass ordering, $m_{\tilde{\eta}^{\prime}}<m_{\tilde{\pi}} \sim m_{\tilde{K}}<m_{\tilde{\eta}}$ [16], at high density, turns into the normal mass ordering, $m_{\pi}<m_{K}<m_{\eta}<m_{\eta^{\prime}}$, at low density.

\section{Acknowledgments}

We would like to thank Mark Alford for helpful discussions and comments. This research was supported in part by the Grants-in-Aid of the Japanese Ministry of Education, Culture, Sports, Science, and Technology (No. 18540253), and in part by NSF Grant PHY03-55014. Author GB thanks the University of Tokyo for its kind hospitality as well as support through the COE. 


\section{APPENDIX A: DERIVATION OF PHASE BOUNDARIES AND CRITICAL POINTS}

In this Appendix we describe more quantitatively the phase boundaries for three and two massless flavors respectively for both a positive and negative fourth order term, $b>0$ or $b<0$. We only outline the derivation of the phase boundaries and critical points; the results can be confirmed explicitly by comparing the free energies.

\section{Three massless flavors}

To find the minimum of the GL free energy $\Omega_{3 F}$ in Eq. (17), it is useful to eliminate either of the variables $\sigma$ or $d$ by solving the stationarity condition,

$$
\frac{\partial \Omega_{3 F}}{\partial d}=2 d\left(\frac{\alpha}{2}+\frac{\beta}{2} d^{2}-\gamma \sigma\right)=0
$$

which yields $d=0$ and $d^{2}=(2 / \beta)(\gamma \sigma-\alpha / 2)$. The phase boundaries and the order of the phase transitions are obtained by comparing the free energies: $\Omega^{(\mathrm{CSC})}(0, d)\left(=\alpha d^{2} / 2+\beta d^{4} / 4\right), \Omega^{(\mathrm{NG})}(\sigma, 0)\left(=a \sigma^{2} / 2-c \sigma^{3} / 3+b \sigma^{4} / 4+f \sigma^{6} / 6\right)$, and $\Omega^{(\mathrm{COE})}$ measured with respect to that of the normal phase, $\Omega^{(\mathrm{NOR})}(0,0)$. Using the $d \neq 0$ solution of Eq. (A1), we have

$$
\Omega^{(\mathrm{COE})}(\sigma, d(\sigma))=-\frac{\alpha^{2}}{4 \beta}+\gamma^{*} \sigma+\frac{a^{*}}{2} \sigma^{2}-\frac{c}{3} \sigma^{3}+\frac{b}{4} \sigma^{4}+\frac{f}{6} \sigma^{6} \quad\left(\sigma \geq \frac{\alpha}{2 \gamma}\right)
$$

with

$$
\begin{aligned}
& \gamma^{*} \equiv \alpha \gamma / \beta, \\
& a^{*} \equiv a-2 \gamma^{2} / \beta,
\end{aligned}
$$

or equivalently,

$$
\Omega^{(\mathrm{COE})}(\sigma(d), d)=\Omega_{0}+\frac{\alpha^{*}}{2} d^{2}+\frac{\beta^{*}}{4} d^{4}+\text { higher order terms }
$$

with

$$
\begin{aligned}
\Omega_{0} & \equiv \frac{\alpha^{2}}{384 \gamma^{6}}\left(48 \gamma^{4} a-16 \gamma^{3} c \alpha+6 \gamma^{2} b \alpha^{2}+f \alpha^{4}\right), \\
\alpha^{*} & \equiv \frac{\beta \alpha}{32 \gamma^{6}}\left(f \alpha^{4}+4 \gamma^{2} b \alpha^{2}-8 \gamma^{3} c \alpha+16 \gamma^{4} a\right), \\
\beta^{*} & \equiv \frac{\beta^{2}}{32 \gamma^{6}}\left(5 f \alpha^{4}+12 \gamma^{2} b \alpha^{2}-16 \gamma^{3} c \alpha+16 \gamma^{4} a-32 \frac{\gamma^{6}}{\beta}\right) .
\end{aligned}
$$

\section{a. Positive $b$}

For $b>0$, the sixth order $f$ term in Eq. (17) does not change the qualitative structure of the phase diagram and can be safely neglected. In the following, we study the phase boundaries ABCC', BB', CD, and CDD' in Fig. 2 separately and find the locations of the characteristic points A, B, C and D.

- The critical point A and the first order phase boundary ABCC'

We first eliminate the $\sigma^{3}$ term in Eq. (A2) for the COE phase by introducing a new field $\tau=\sigma-c /(3 b)$, in terms of which,

$$
\Omega^{(\mathrm{COE})}(\tau)=\Omega_{\mathrm{c}}+\gamma_{\mathrm{c}}^{*} \tau+\frac{a_{\mathrm{c}}^{*}}{2} \tau^{2}+\frac{b}{4} \tau^{4}
$$


with

$$
\begin{aligned}
& \Omega_{\mathrm{c}}=-\frac{\beta}{4 \gamma^{2}} \gamma^{* 2}+\frac{c}{3 b} \gamma^{*}+\frac{c^{2}}{18 b^{2}} a^{*}-\frac{c^{4}}{108 b^{2}}, \\
& \gamma_{\mathrm{c}}^{*}=\gamma^{*}+\frac{c}{3 b} a^{*}-\frac{2 c^{3}}{27 b^{2}}, \\
& a_{\mathrm{c}}^{*}=a^{*}-\frac{c^{2}}{3 b},
\end{aligned}
$$

where $a^{*}$ and $\gamma^{*}$ are given in Eqs. (A3) and (A4). The system described by Eq. (A99) is equivalent to an Ising ferromagnet in an external magnetic field. Thus the point A corresponds to the second order critical point of the equivalent magnetic system at $\gamma_{\mathrm{c}}^{*}=a_{\mathrm{c}}^{*}=0$. The location of $\mathrm{A}$ in terms of the original coordinates is:

$$
\mathrm{A}=\left(\frac{c^{2}}{3 b}+\frac{2 \gamma^{2}}{\beta},-\frac{\beta c^{3}}{27 \gamma b^{2}}\right)
$$

thus $\mathrm{A}$ is always located in the region $a>0$ and $\alpha<0$, as shown in Fig. 2 The first order line AB in the COE phase, determined by the condition $\gamma_{\mathrm{c}}^{*}=0$,

$$
\mathrm{AB}: \alpha=-\frac{\beta c}{3 \gamma b} a+\frac{2 \gamma c}{3 b}+\frac{2 \beta c^{3}}{27 \gamma b^{2}}
$$

is straight with negative slope, as shown in Fig. 2. For negative $\alpha, \tau$ jumps discontinuously across $\mathrm{AB}$ between the solutions in the COE phase, $\tau= \pm \sqrt{-a_{\mathrm{c}}^{*} / b}$. For positive $\alpha$, there is a jump between the COE phase and the NOR phase as shown below. Thus the coordinates of the point $\mathrm{B}$ are

$$
\mathrm{B}=\left(\frac{2 c^{2}}{9 b}+\frac{2 \gamma^{2}}{\beta}, 0\right)
$$

The line $\mathrm{BC}$, obtained from $\Omega^{(\mathrm{COE})}=\Omega^{(\mathrm{NOR})}=0$ at the potential minimum, is first order, since the bracket in Eq. (A1) always vanishes in the COE phase and either $\sigma$ or $d$ must be discontinuous from the NOR phase to the COE phase. The expression for $\mathrm{BC}$ is easy to derive but is too complicated to show here. For sufficiently large $\alpha$, the COE phase turns into the NG phase, as shown below. The boundary CC' separating the NG phase and the NOR phase is not affected by the coupling $\gamma$ since $d$ vanishes in both phases. Then the condition $\Omega^{(\mathrm{NG})}=\Omega^{(\mathrm{NOR})}=0$ at the potential minimum implies

$$
\mathrm{CC}^{\prime}: a=\frac{2 c^{2}}{9 b}
$$

- The phase boundaries BB' and CD'

To determine the boundaries primarily associated with the diquark condensate, it is most useful to study the reduced free energy $\Omega^{(\mathrm{COE})}(\sigma(d), d)$, Eq. (A5). When $\beta^{*}$ is positive, the system is equivalent to a usual Ising ferromagnet and shows a second order transition at $\alpha^{*}=0$. One finds two boundaries,

$$
\begin{array}{ll}
\mathrm{BB}^{\prime} & : \alpha=0, \\
\mathrm{CD}^{\prime} & : \alpha=\frac{\gamma}{b}\left(c+\sqrt{c^{2}-4 b a}\right) .
\end{array}
$$

We have chosen the larger root of $b \alpha^{2}-2 \gamma c \alpha+4 \gamma^{2} a=0$ obtained from $\alpha^{*}=0$ in Eq. (A16) as the only root compatible with the condition that $\Omega^{(\mathrm{COE})}=\Omega^{(\mathrm{NG})} \leq \Omega^{(\mathrm{NOR})}$. Thus the coordinates of the critical end point $\mathrm{C}$ are

$$
\mathrm{C}=\left(\frac{2 c^{2}}{9 b}, \frac{4 c \gamma}{3 b}\right) \text { for } \beta^{*}>0
$$

The condition $\beta^{*}>0$ on the line CD' is equivalent to $0<\gamma<(c / 3) \sqrt{\beta / b}$. If $\beta^{*}<0$, or equivalently $\gamma>(c / 3) \sqrt{\beta / b}$, a tricritical point D determined by $\alpha^{*}=\beta^{*}=0$ appears on CD', as shown in the right panel of Fig. 22 In this case, the line $\mathrm{CD}$ becomes first order and $\mathrm{C}$ becomes a triple point. The location of $\mathrm{D}$ is

$$
\mathrm{D}=\left(\frac{c\left(c+c^{\prime}\right)}{8 b}-\frac{\gamma^{2}}{\beta}, \frac{\gamma\left(c+c^{\prime}\right)}{2 b}\right)
$$


with $c^{\prime} \equiv \sqrt{c^{2}+16 b \gamma^{2} / \beta}$. The point $\mathrm{C}$ is determined by the coexistence of the three phases, COE, NG, and NOR, so that the free energies $\Omega^{(\mathrm{COE})}=\Omega^{(\mathrm{NG})}$ measured with respect to the normal phase, vanish at their minima; then

$$
\mathrm{C}=\left(\frac{2 c^{2}}{9 b}, \sqrt{\frac{\beta}{b}}\left(\frac{c}{3 \sqrt{b}}+\frac{\gamma}{\sqrt{\beta}}\right)^{2}\right) \quad \text { for } \beta^{*}<0 .
$$

\section{b. Negative $b$}

For $b<0$, the presence of the sixth order $f$-term in Eq. (17) is crucial. Nonetheless, the topological structure of the phase diagram in Fig. 2 is exactly the same as for $b>0$.

- The critical point A and the first order phase boundary ABCC'

With $b<0$ and the $f$ term, the free energy $\Omega^{(\mathrm{COE})}(\sigma, d(\sigma))$ in the COE phase has at most three local minima, which we label $\sigma_{i}(i=1,2,3)$. The competition among the three phases leads to at most three first order lines. However, the detailed stability analysis outlined below show that there is only one first order line and critical point in the COE phase. Thus the phase structure for $b<0$ is the same as that for $b>0$.

Let us now determine the two competing chiral condensates in the COE phase, labelled by $\sigma_{1}$ and $\sigma_{2}$, at which $\Omega_{1}^{(\mathrm{COE})}=\Omega_{2}^{(\mathrm{COE})}=\Omega_{\min }$. Then $\Omega^{(\mathrm{COE})}(\sigma, d(\sigma))=-\alpha^{2} / 4 \beta+\gamma^{*} \sigma+a^{*} \sigma^{2} / 2-c \sigma^{3} / 3+b \sigma^{4} / 4+f \sigma^{6} / 6=(f / 6)(\sigma-$ $\left.\sigma_{1}\right)^{2}\left(\sigma-\sigma_{2}\right)^{2}\left(\sigma-\tau_{1}\right)\left(\sigma-\tau_{2}\right)+\Omega_{\min }$ where $\tau_{1,2}$ are complex except when $\tau_{1}=\tau_{2}=\sigma_{3}$. Eliminating $\tau_{1,2}$ and defining $r \equiv \sigma_{1}+\sigma_{2}$ and $s \equiv \sigma_{1} \sigma_{2}$, we have

$$
4 f r^{3}+(3 b-6 f s) r-2 c=0
$$

and

$$
\begin{aligned}
a^{*} & =f r^{4}+\frac{b}{2} r^{2}-f s^{2}+b s, \\
\gamma^{*} & =-s r\left(f r^{2}-f s+\frac{b}{2}\right), \\
\Omega_{\min } & =-\frac{f}{6}\left(2 s-3 r^{2}\right) s^{2}-\frac{b}{4}-\frac{\alpha^{2}}{4 \beta} .
\end{aligned}
$$

In the COE phase, several conditions must be fulfilled: (i) $\sigma_{1,2} \geq \alpha / 2 \gamma$, (ii) $\Omega_{1}=\Omega_{2} \leq \Omega_{3}$, (iii) $\Omega^{(\mathrm{COE})} \leq \Omega^{(\mathrm{NOR})}$, and (iv) $\Omega^{(\mathrm{COE})} \leq \Omega^{(\mathrm{NG})}$. From Eq. (A20), we derive a necessary and sufficient condition for (i)-(iv), $0 \leq 2 \sqrt{s} \leq r$. Under this condition, Eq. (A20), a cubic equation in terms of $r$, has only one root satisfying $r_{\chi} \leq r \leq r_{\mathrm{c}}$. The upper limit $r_{\chi}$ and lower limit $r_{\mathrm{c}}$, and correspondingly $s_{\chi}$ and $s_{\mathrm{c}}$, satisfy

$$
\begin{aligned}
& 5 f r_{\mathrm{c}}^{3}+6 b r_{\mathrm{c}}-4 c=0, \quad s_{\mathrm{c}}=4 r_{\mathrm{c}}^{2}, \\
& 4 f r_{\chi}^{3}+3 b r_{\chi}-2 c=0, \quad s_{\chi}=0 .
\end{aligned}
$$

The root $r=r_{\mathrm{c}}$ corresponds to the critical point A since the two minima merge into one, $\sigma_{1}=\sigma_{2}$, while $r=r_{\chi}$ corresponds to the point B where the two local minima are $\sigma_{1}=0$ and $\sigma_{2} \neq 0$. Using Eqs. (A20), (A21) and (A22), we thus find

$$
\begin{aligned}
\mathrm{A} & =\left(-\frac{3}{8} b r_{\mathrm{c}}^{2}+\frac{3}{4} c r_{\mathrm{c}}+\frac{2 \gamma^{2}}{\beta}, \frac{\beta}{20 \gamma}\left(2 b r_{\mathrm{c}}^{3}-3 c r_{\mathrm{c}}^{2}\right)\right), \\
\mathrm{B} & =\left(-\frac{b}{4} r_{\chi}^{2}+\frac{c}{2} r_{\chi}+\frac{2 \gamma^{2}}{\beta}, 0\right) .
\end{aligned}
$$

It can be shown that the point $\mathrm{A}$ is always in the fourth quadrant $(a>0$ and $\alpha<0)$ and that the point $\mathrm{B}$ is always on the $a$-axis.

The line $\mathrm{AB}$ located in $\alpha \leq 0$ corresponds to the interval, $r_{\chi} \leq r \leq r_{\mathrm{c}}$. Thus there is only one first order line, $\mathrm{AB}$, and critical point, $\mathrm{A}$, in the $\mathrm{COE}$ phase. The line $\mathrm{BC}$, whose explicit form is complicated, is first order, as for $b>0$, since Eq. (A1) is always satisfied in the COE phase and either $\sigma$ or $d$ is discontinuous across BC. The boundary CC' is not affected by the diquark condensate and, following the similar argument leading to Eq. (A20), we have

$$
\mathrm{CC}^{\prime}: a=-\frac{b}{4} r_{\chi}^{2}+\frac{c}{2} r_{\chi}
$$


which is in the region $a>0$.

- Phase boundaries BB' and CD'

As for $b>0$, the lines $\mathrm{BB}^{\prime}$ and $\mathrm{CD}^{\prime}$ obtained from $\alpha^{*}=0$ are

$$
\begin{array}{ll}
\mathrm{BB}^{\prime}: & \alpha=0, \\
\mathrm{CD}^{\prime}: & f \alpha^{4}+4 \gamma^{2} b \alpha^{2}-8 \gamma^{3} c \alpha+16 \gamma^{4} a=0 .
\end{array}
$$

The maximum root of $\alpha$ in Eq. (A28) should be chosen to satisfy the condition on CD': $\Omega^{(\mathrm{COE})}=\Omega^{(\mathrm{NG})} \leq \Omega^{(\mathrm{NOR})}$. The coordinates of $\mathrm{C}$ for $\beta^{*}>0$ are easily found from the crossing of CD' and CC':

$$
\mathrm{C}=\left(-\frac{b}{4} r_{\chi}^{2}+\frac{c}{2} r_{\chi}, 2 \gamma r_{\chi}\right), \text { for } \beta^{*}>0
$$

If $f<b^{3}\left(c-3 c^{\prime \prime}\right) /\left(c+c^{\prime \prime}\right)^{3}$ with $c^{\prime \prime} \equiv \sqrt{c^{2}-8 b \gamma^{2} / \beta}, \beta^{*}$ can change sign on the line CD', and a tricritical point D determined by $\alpha^{*}=\beta^{*}=0$ appears, as shown in the right panel of Fig. 2. In this case, the line CD becomes first order and the $\mathrm{C}$ becomes a triple point.

Thus we find that the topological phase structure in the massless three-flavor system with $\gamma>0$ and $\lambda=0$ is independent of the sign of $b$, and a critical point A always appears in the COE phase as shown in Fig. 2.

\section{c. Effect of small $\lambda$}

Finally, we discuss the effect of the term $\lambda d^{2} \sigma^{2}$, hitherto neglected. With this term, the stationarity condition becomes [cf. Eq. (A1)],

$$
\frac{\partial \Omega_{3 F}}{\partial d^{2}}=\frac{\alpha}{2}+\frac{\beta}{2} d^{2}-\gamma \sigma+\lambda \sigma^{2}=0
$$

Accordingly, the reduced free-energy $\Omega^{(\mathrm{COE})}(\sigma, d(\sigma))$ in Eq. (A2) is modified as

$$
\Omega^{(\mathrm{COE})}(\sigma, d(\sigma))=-\frac{\alpha^{2}}{4 \beta}+\gamma^{*} \sigma+\frac{a_{\lambda}^{*}}{2} \sigma^{2}-\frac{c_{\lambda}}{3} \sigma^{3}+\frac{b_{\lambda}}{4} \sigma^{4}+\frac{f}{6} \sigma^{6},
$$

where the new coefficients are

$$
a_{\lambda}^{*}=a^{*}-2 \alpha \frac{\lambda}{\beta}, \quad c_{\lambda}=c-6 \gamma \frac{\lambda}{\beta}, \quad b_{\lambda}=b-4 \lambda \frac{\lambda}{\beta} .
$$

Therefore, insofar as $|\lambda / \beta| \ll 1$, as is suggested microscopically (see Appendix $\mathbf{C}$ ), the $\lambda$ term does not qualitatively change the phase diagram in Fig. 2

\section{Two massless flavors}

Following the same argument as in the three flavor case, we use the stationarity condition for the GL free energy $\Omega_{2 F}$ in Eq. (21),

$$
\frac{\partial \Omega_{2 F}}{\partial d}=2 d\left(\frac{\alpha}{2}+\frac{\beta}{2} d^{2}+\lambda \sigma^{2}\right)=0
$$

to obtain the reduced free-energies:

$$
\Omega^{(\mathrm{COE})}(\sigma, d(\sigma))=-\frac{\alpha^{2}}{4 \beta}+\frac{a^{\prime}}{2} \sigma^{2}+\frac{b^{\prime}}{4} \sigma^{4}+\frac{f}{6} \sigma^{6},
$$

for $\sigma^{2} \leq-\alpha /(2 \lambda)$, and

$$
\Omega^{(\mathrm{COE})}(\sigma(d), d)=\Omega_{0}+\frac{\alpha^{\prime}}{2} d^{2}+\frac{\beta^{\prime}}{4} d^{4}-\frac{f \beta^{3}}{48 \lambda^{3}} d^{6},
$$


for $d^{2} \leq-\alpha / \beta$. Here the coeficients are

$$
\begin{aligned}
a^{\prime} & \equiv a-2 \lambda \alpha / \beta, \\
b^{\prime} & \equiv b-4 \lambda^{2} / \beta, \\
\Omega_{2 F}^{0} & \equiv \alpha\left(-12 \lambda^{2} a+3 \lambda b \alpha-f \alpha^{2}\right) /\left(48 \lambda^{3}\right), \\
\alpha^{\prime} & \equiv-\left(f \alpha^{2}-2 \lambda b \alpha+4 \lambda^{2} a\right) \beta^{2} /\left(8 \lambda^{3}\right), \\
\beta^{\prime} & \equiv\left(b^{\prime} \lambda-f \alpha\right) \beta^{2} /\left(4 \lambda^{2}\right) .
\end{aligned}
$$

Note that the potential (A35) is bounded, despite the negative $d^{6}$ term, a consequence of the condition $d^{2} \leq-\alpha / \beta$.

\section{a. positive $b$}

As in an Ising ferromagnet, the phase boundaries, between NOR and NG, and NOR and CSC, for $b>0$ and $f=0$ are characterized by second order lines, $a=0$ and $\alpha=0$, respectively. For both $\beta^{\prime}>0$ and $b^{\prime}>0$, or equivalently,

$$
\frac{1}{2} \sqrt{b \beta}>\lambda>0,
$$

the COE-NG and COE-CSC phase boundaries are characterized by $\alpha^{\prime}=0$ and $a^{\prime}=0$, respectively. These conditions can be rewritten as

$$
\begin{array}{ll}
\alpha=\frac{2 a \lambda}{b}, & \text { COE }- \text { NG boundary }, \\
\alpha=\frac{a \beta}{2 \lambda}, & \text { COE }- \text { CSC boundary },
\end{array}
$$

depicted in the left panel of Fig. 3 .

On the other hand, when $\lambda>\sqrt{b \beta} / 2$, the COE phase ceases to exist. Then the competition between the free energies $\Omega_{\min }^{(\mathrm{NG})}=-a^{2} /(4 b)$ and $\Omega_{\mathrm{min}}^{(\mathrm{CSC})}=-\alpha^{2} /(4 \beta)$ determines the first order NG-CSC boundary:

$$
\alpha=a \sqrt{\beta / b}
$$

as shown in the right panel of Fig. 3 .

\section{b. negative $b$}

The case $b<0$ requires introduction of a positive $f$ term.

- First-order phase boundary E'EFF'

The phase boundaries EE' and FF' are determined by $\Omega^{(\mathrm{NG})}=\Omega^{(\mathrm{NOR})}$ and $\Omega^{(\mathrm{COE})}=\Omega^{(\mathrm{CSC})}$ at the potential minimum,

$$
\begin{array}{ll}
\mathrm{EE}^{\prime}: & a=3 b^{2} /(16 f) . \\
\mathrm{FF}^{\prime}: & \alpha=\frac{\beta}{2 \lambda}\left[a-3\left(b-4 \lambda^{2} / \beta\right)^{2} /(16 f)\right] .
\end{array}
$$

To determine the boundary EF, we use the free-energies of the NG and CSC phases,

$$
\begin{aligned}
\Omega_{\min }^{(\mathrm{NG})} & =-\left[6 b f a+\left(b^{2}-4 f a\right)^{3 / 2}-b^{3}\right] /\left(24 f^{2}\right), \\
\Omega_{\min }^{(\mathrm{CSC})} & =-\alpha^{2} /(4 \beta),
\end{aligned}
$$

where the global minima at $\sigma \neq 0$ in the NG phase and $d \neq 0$ in the CSC phase implicitly ensure $b^{2}-4 f a \geq 0$ $(\alpha \leq 0)$. From $\Omega_{\mathrm{min}}^{(\mathrm{NG})}=\Omega_{\mathrm{min}}^{(\mathrm{CSC})}$, we obtain

$$
\mathrm{EF}: \alpha=-\left\{\beta\left[6 b f a+\left(b^{2}-4 f a\right)^{3 / 2}-b^{3}\right] /\left(6 f^{2}\right)\right\}^{1 / 2} .
$$


The critical end points $\mathrm{E}$ and $\mathrm{F}$ are then

$$
\mathrm{E}=\left(\frac{3 b^{2}}{16 f}, 0\right), \quad \mathrm{F}=\left(\frac{3 b^{\prime}}{16 f}\left(b^{\prime}+\frac{16 \lambda^{2}}{\beta}\right), \frac{3 \lambda b^{\prime}}{2 f}\right) .
$$

- Second order phase boundary EG' and FH'

The boundary EG' is not affected by the chiral condensate and is characterized by $\alpha=0$, while $\mathrm{FH}^{\prime}$ is given by $\alpha^{\prime}=0$ :

$$
\begin{aligned}
& \text { EG' }: \alpha=0, \\
& \text { FH' }^{\prime}: \alpha=\frac{\lambda}{f}\left(b-\sqrt{b^{2}-4 f a}\right) .
\end{aligned}
$$

These boundaries indeed terminate at the points $\mathrm{E}$ and $\mathrm{F}$ given in Eq. (A45). On EG' and FH', the coefficient $\beta^{\prime}$ never changes sign, and therefore a tricritical point does not appear, unlike in the three-flavor case (the point $\mathrm{D}$ in the right panel of Fig. 2).

While we have left out details of the derivation of the phase structure in Fig. 4, the structure can, as mentioned earlier, be explicitly checked by direct comparison of the free energies.

\section{APPENDIX B: SIGN OF $\gamma$ FOR THREE FLAVORS}

In this Appendix we summarize how the anomaly-induced interaction $-\gamma d^{2} \sigma$ in Eq. (17) results from the basic 6-Fermi interaction. The latter has the form [2]

$$
\mathcal{H}_{\text {inst }}=-\mathcal{L}_{\text {inst }}=-g_{\mathrm{D}}\left[\operatorname{det}_{i, j} \bar{q}_{a}^{i}\left(1-\gamma_{5}\right) q_{a}^{j}+\text { h.c. }\right]=-2 g_{\mathrm{D}}\left[\operatorname{det}_{i, j} \bar{q}_{R a}^{i} q_{L a}^{j}+\text { h.c. }\right],
$$

with $g_{\mathrm{D}}<0$. The final $\left(c_{0}\right)$ term of the GL free energy in Eq. (7) originates from the expectation value (in lowest order) of $\mathcal{H}_{\text {inst }}$; inserting $\Phi$ from Eq.(41) into Eq.(7), we have

$$
\Omega_{\mathrm{inst}}=\frac{c_{0}}{2} G_{\chi}^{3}\left\langle\operatorname{det}_{i, j}\left(\bar{q}_{R a}^{i} q_{L a}^{j}\right)+\text { h.c. }\right\rangle=\frac{c_{0}}{2} \cdot G_{\chi}^{3} \cdot \frac{1}{6} \cdot \epsilon_{i j k} \epsilon_{i^{\prime} j^{\prime} k^{\prime}}\left\langle\left(\bar{q}_{R a}^{i} q_{L a}^{i^{\prime}}\right)\left(\bar{q}_{R b}^{j} q_{L b}^{j^{\prime}}\right)\left(\bar{q}_{R c}^{k} q_{L c}^{k^{\prime}}\right)\right\rangle+\text { c.c.. }
$$

Thus the GL coefficient is related to the microscopic coupling by $c_{0} G_{\chi}^{3} / 2=-2 g_{\mathrm{D}}>0$. The coefficient $c_{0}$ must be positive in order for $m_{\eta^{\prime}}^{2} \sim c_{0}$ to be positive (see Eq. (D1)). In addition, for $c_{0}>0$, the chiral condensate at low temperature is positive. We have $G_{\chi}>0$ as well.

To proceed, we replace one of the $\bar{q}_{R} q_{L}$ pairs in Eq. (B2) by the chiral field $\Phi$, which gives

$$
\begin{aligned}
\Omega_{\mathrm{inst}} & \simeq-\frac{c_{0}}{2} \cdot G_{\chi}^{2} \cdot \frac{3}{6} \cdot \epsilon_{i j k} \epsilon_{i^{\prime} j^{\prime} k^{\prime}}\left\langle\left(\bar{q}_{R a}^{i} q_{L a}^{i^{\prime}}\right)\left(\bar{q}_{R b}^{j} q_{L b}^{j^{\prime}}\right)\right\rangle \Phi_{k^{\prime} k}+\text { c.c. } \\
& \simeq \frac{c_{0}}{2} \cdot G_{\chi}^{2} \cdot \frac{3}{6} \cdot \frac{2}{4} \cdot \epsilon_{i j k} \epsilon_{i^{\prime} j^{\prime} k^{\prime}}\left\langle\left(\bar{q}_{R a}^{i} C \bar{q}_{R b}^{j}\right)\left(q_{L b}^{j^{\prime}} C q_{L a}^{i^{\prime}}\right)\right\rangle \Phi_{k^{\prime} k}+\text { c.c. } \\
& =c_{0}\left(\frac{G_{\chi}}{G_{d}}\right)^{2} \operatorname{Tr}\left[\left(d_{R} d_{L}^{\dagger}\right) \Phi\right]+\text { c.c. } \equiv \gamma_{1} \operatorname{Tr}\left[\left(d_{R} d_{L}^{\dagger}\right) \Phi\right]+\text { c.c.. }
\end{aligned}
$$

In deriving (B4) from (B3) we use the fermion anti-commutator twice and the Fierz transform; The overall sign changes from - to + because the coefficient of the $C C$ term in the Fierz transform is negative. Then in deriving (B5) from (B4) we use the definition of the diquark condensate, Eq. (8). The contraction of the $\epsilon$-tensor three-times gives $+(-)^{2}$ preserving the overall sign. The coefficient $\gamma_{1}$, defined in Eq. (14) is thus $c_{0}\left(G_{\chi} / G_{d}\right)^{2}>0$.

With the CFL ansatz (16), the anomaly-induced term reduces to

$$
\Omega_{\text {inst }} \rightarrow-3 \gamma_{1} d^{2} \sigma \equiv-\gamma d^{2} \sigma
$$

The minus sign originates from $d_{L}=-d_{R}$. Thus we finally find that $\gamma$ [Eq. (17)] is given by

$$
\gamma=3 \gamma_{1}=3 c\left(\frac{G_{\chi}}{G_{d}}\right)^{2}>0
$$

This result is consistent with a similar result given in [17], where it is found that the effect of the quark mass combined with the axial anomaly reduces the energy of the CFL phase. 


\section{APPENDIX C: $\lambda, \beta$ AND $b$ FROM THE NJL MODEL}

The $\lambda$ in Eq. (17) may be evaluated in the three-flavor and two-flavor NJL models as follows. We start with the free energy Eq. (5.15) in Ref. [8] for three flavors, and Eq. (4.50) for two flavors, in the chiral limit:

$$
\begin{aligned}
& \Omega_{3 F}(\sigma, d)=\frac{3 \sigma^{2}}{2 G_{\chi}}-c_{0} \sigma^{3}+\frac{3 d^{2}}{2 G_{d}}-\int \frac{d^{3} p}{(2 \pi)^{3}} \sum_{ \pm}\left[8 \omega_{8}^{ \pm}+\omega_{1}^{ \pm}+16 T \ln \left(1+e^{-\omega_{8}^{ \pm} / T}\right)+2 T \ln \left(1+e^{-\omega_{1}^{ \pm} / T}\right)\right],(C) \\
& \Omega_{2 F}(\sigma, d)=\frac{\sigma^{2}}{2 G_{\chi}}+\frac{d^{2}}{2 G_{d}}-\int \frac{d^{3} p}{(2 \pi)^{3}} \sum_{ \pm}\left[4 \omega^{ \pm}+2 E^{ \pm}+8 T \ln \left(1+e^{-\omega^{ \pm} / T}\right)+4 T \ln \left(1+e^{-E^{ \pm} / T}\right)\right]
\end{aligned}
$$

where

$$
\begin{aligned}
E^{ \pm} & =\sqrt{p^{2}+M^{2}} \pm \mu \text { with } M=\left\{\begin{array}{cc}
\sigma-c_{0} G_{\chi} \sigma^{2} / 2 & \text { (three flavors) } \\
\sigma & \text { (two flavors) }
\end{array}\right. \\
\omega_{8(1)}^{ \pm} & =\sqrt{\left(E^{ \pm}\right)^{2}+d_{8(1)}^{2}}, \\
\omega^{ \pm} & =\sqrt{\left(E^{ \pm}\right)^{2}+d^{2}} .
\end{aligned}
$$

Here $d_{8}=d$ is the octet gap, and $d_{1}=2 d_{8}$ the singlet gap, for nine quasi-quarks (three colors times three flavors). The $c_{0}$-term originates from the instanton-induced interaction; we ignore in Eq. (C1) the contribution from the quark-quark and quark-anti-quark six-point instanton-induced interaction (the $\gamma_{1}$-term in Eq. (14)). Since the fermion integral does not diverge in the infrared, a naive power-series expansion of $\Omega$ in $d$ and $\sigma$ should be valid.

The gap is determined by minimizing $\Omega$ with respect to $d^{2}$ :

$$
\begin{aligned}
& \frac{\partial \Omega_{3 F}}{\partial d^{2}}=\frac{3}{2 G_{d}}-\sum_{ \pm} \int \frac{d^{3} p}{(2 \pi)^{3}}\left\{\frac{4}{\omega_{8}^{ \pm}} \tanh \frac{\omega_{8}^{ \pm}}{2 T}+\frac{2}{\omega_{1}^{ \pm}} \tanh \frac{\omega_{1}^{ \pm}}{2 T}\right\}, \\
& \frac{\partial \Omega_{2 F}}{\partial d^{2}}=\frac{1}{2 G_{d}}-\sum_{ \pm} \int \frac{d^{3} p}{(2 \pi)^{3}}\left\{\frac{2}{\omega^{ \pm}} \tanh \frac{\omega^{ \pm}}{2 T}\right\},
\end{aligned}
$$

where we introduce an ultraviolet cutoff at $p=\Lambda$ to regulate the integrals. In the NJL model, $\Lambda \simeq 1 \mathrm{GeV}$, while in weakcoupling QCD at high density, the momentum dependence of the gap function leads to $\Lambda \simeq 256 \pi^{4}\left(2 / 3 g^{2}\right)^{5 / 2} \mu \gg \mu$ [15]. In the following, we always assume $\Lambda \gg \mu$ and $\Lambda \gg T$. The - terms are the contribution from the particles and the + terms from anti-particles. The critical temperature $T_{c}$ of the super-to-normal transition for three flavors is determined by setting the right side of Eq. (C6), and of Eq. (C7) for two flavors, to zero.

The term $\lambda \sigma^{2} d^{2}$ in the GL expansion of $\Omega(\sigma, d)$ is obtained as

$$
\lambda_{3 F}=\left.\frac{\partial^{2} \Omega_{3 F}}{\partial \sigma^{2} \partial d^{2}}\right|_{\sigma=d=0}=-\frac{3}{2 \pi^{2}} \sum_{ \pm} \int_{0}^{\Lambda} d p p \frac{\partial}{\partial p}\left[\frac{1}{p \pm \mu} \tanh \frac{(p \pm \mu)}{2 T}\right] .
$$

Evaluating the above integral by partial integration, making the approximation, $\tanh x \simeq 1(x>1)$, tanh $x \simeq-1(x<$ $-1)$ and $\tanh x \simeq x(-1 \leq x \leq 1)$, we arrive at

$$
\lambda_{3 F} \simeq \frac{3}{2 \pi^{2}} \ln \left(\frac{\Lambda}{2 T}\right)^{2}>0, \quad \lambda_{2 F}=\lambda_{3 F} / 3 .
$$

We can similarly calculate the coefficient $\beta$ in the term $\beta\left(d^{2}\right)^{2} / 4$. For three flavors

$$
\beta_{3 F}=\left.2 \frac{\partial^{2} \Omega_{3 F}}{\partial d^{2} \partial d^{2}}\right|_{\sigma=d=0}=-\frac{6}{\pi^{2}} \sum_{ \pm} \int_{0}^{\Lambda} d p \frac{p^{2}}{p \pm \mu} \frac{\partial}{\partial p}\left[\frac{1}{p \pm \mu} \tanh \frac{(p \pm \mu)}{2 T}\right]
$$

which leads to

$$
\beta_{3 F} \simeq \frac{6}{\pi^{2}}\left[\left(\frac{\mu}{2 T}\right)^{2}+\ln \left(\frac{\Lambda}{2 T}\right)^{2}\right]>0, \quad \beta_{2 F}=\beta_{3 F} / 6
$$

For typical values in the NJL model at intermediate density: $\Lambda=1 \mathrm{GeV}, \mu=500 \mathrm{MeV}$ and $T=50 \mathrm{MeV}$, we have $\lambda_{3 F} / \beta_{3 F} \simeq 0.7 / 18=0.04$. Also, at asymptotically high density in QCD with $\Lambda \propto \mu$, we have $\lambda_{3 F} / \beta_{3 F} \sim$ $\ln (\mu / T)^{2} /(\mu / T)^{2}$ which is parametrically small at large $\mu$. 
The coefficient $b$ in the term $b\left(\sigma^{2}\right)^{2} / 4$ can be calculated from the free energy Eq. (C1), or Eq. (C2), with $d=0$ :

$$
b_{N_{f}}=\left.2 \frac{\partial^{2} \Omega_{N_{f}}}{\partial \sigma^{2} \partial \sigma^{2}}\right|_{\sigma=0}=-\frac{3 N_{f}}{4 \pi^{2}} \sum_{ \pm} \int_{0}^{\Lambda} d p p \frac{\partial}{\partial p}\left[\frac{1}{p} \tanh \frac{(p \pm \mu)}{2 T}\right],
$$

where we have assumed $M=\sigma$ for both two and three flavors, for simplicity. Evaluating the integral, we find

$$
b_{N_{f}} \simeq \frac{3 N_{f}}{4 \pi^{2}}\left[\left(1+\frac{\mu}{2 T}\right) \ln \left|\frac{\Lambda / 2 T}{1+\mu / 2 T}\right|+\left(1-\frac{\mu}{2 T}\right) \ln \left|\frac{\Lambda / 2 T}{1-\mu / 2 T}\right|\right] .
$$

Unlike for the coefficients $\lambda$ and $\beta$, the sign of $b$ changes from positive to negative as $\mu$ increases from zero.

Finally, we show the integral form of the coefficient $\tilde{b}_{2 F}$ which appears in the term, $\tilde{b}_{2 F} d^{2} \sigma^{4} / 4$ in the GL expansion of the two flavor NJL model:

$$
\tilde{b}_{2 F}=\left.2 \frac{\partial^{3} \Omega_{2 F}}{\partial \sigma^{2} \partial \sigma^{2} \partial d^{2}}\right|_{\sigma=d=0}=-\frac{1}{2 \pi^{2}} \sum_{ \pm} \int_{0}^{\Lambda} d p p^{2}\left(\frac{1}{p} \frac{\partial}{\partial p}\right)^{2}\left[\frac{1}{p \pm \mu} \tanh \frac{(p \pm \mu)}{2 T}\right],
$$

where the sign of $\tilde{b}_{2 F}$ changes, as one can show, from negative to positive as $\mu$ increases from zero.

\section{APPENDIX D: MASS SPECTRA OF THE $\eta^{\prime}$ MESON}

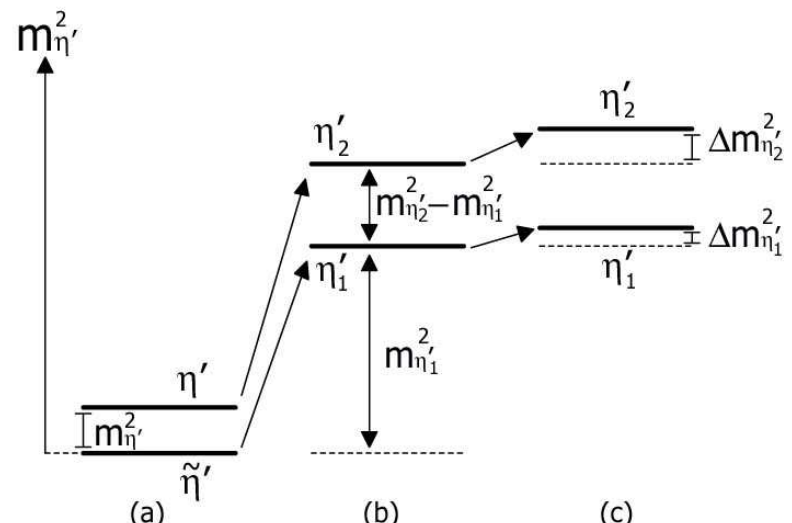

(a)

(b)

(c)

FIG. 7: Mass spectra of $\eta^{\prime}$ meson in the intermediate density region when (a) $\sigma$ - $d$ mixing does not exist $(\gamma=\lambda=0)$; (b) $\sigma-d$ mixing is considered $(\gamma \neq 0, \lambda \neq 0)$; (c) a finite quark mass is considered $\left(m_{q} \neq 0\right)$ in addition. Here $m_{\eta^{\prime}}^{2}=36 c_{0} \sigma^{3} / f_{\eta^{\prime}}^{2}$, $m_{\eta_{1,2}^{\prime}}^{2}\left(m_{q}=0\right)=12\left(x_{1} \mp \sqrt{x_{1}^{2}-x_{2}}\right)$ and $\Delta m_{\eta_{1,2}^{\prime}}^{2}=12\left(x_{3} \mp\left(x_{1} x_{3}-x_{4}\right) / \sqrt{x_{1}^{2}-x_{2}}\right) m_{q}$.

The $\eta^{\prime}$ mass spectra can be found, similarly to that of the pions, by diagonalizing the mass matrix Eq.(151). Figure 7 shows the mass spectra for $\eta^{\prime}$ : (a) with neither the $\sigma$ - $d$ coupling nor a quark mass; (b) with the $\sigma$ - $d$ coupling but without the quark mass; and (c) with both the $\sigma$ - $d$ coupling and non-zero $m_{q}$. In case (a), $\tilde{\eta}^{\prime}$ is massless, since there is no term that breaks the $U(1)_{A}$ symmetry in Eq. (13); on the other hand, $\eta^{\prime}$ is massive because of the axial anomaly, with

$$
m_{\eta^{\prime}}^{2}=36 c_{0} \frac{\sigma^{3}}{f_{\eta^{\prime}}^{2}}
$$

This result differs notably from that for pions, where both $\pi$ and $\tilde{\pi}$ are massless in the absence of the $\sigma$ - $d$ coupling and the quark mass.

With the $\sigma$ - $d$ coupling alone (b), the coupling mixes $\eta^{\prime}$ with $\tilde{\eta}^{\prime}$ and makes the mass eigenstates $\eta_{1,2}^{\prime}$ much heavier than in the absence of the coupling. We have

$$
m_{\eta_{1,2}^{\prime}}^{2}\left(m_{q}=0\right)=12\left(x_{1} \mp \sqrt{x_{1}^{2}-x_{2}}\right)
$$


where

$$
\begin{aligned}
& x_{1}=m_{\eta^{\prime}}^{2}+\frac{\gamma_{1} d^{2} \sigma+\lambda_{3} d^{2} \sigma^{2}}{f_{\eta^{\prime}}^{2}}+\frac{4 \gamma_{1} d^{2} \sigma+\lambda_{3} d^{2} \sigma^{2}}{f_{\tilde{\eta}^{\prime}}^{2}}, \\
& x_{2}=6 \frac{4 c_{0} \gamma_{1} d^{2} \sigma^{4}+c_{0} \lambda_{3} d^{2} \sigma^{5}+6 \gamma_{1} \lambda_{3} d^{4} \sigma^{3}}{f_{\eta^{\prime}}^{2} f_{\tilde{\eta}^{\prime}}^{2}} .
\end{aligned}
$$

The $\gamma_{1}$-term not only mixes the mass spectra of $\eta^{\prime}$ and $\tilde{\eta}^{\prime}$, but also serves itself as a mass term for $\eta^{\prime}$ and $\tilde{\eta}^{\prime}$. On the other hand, the $\lambda_{3}$ term, which preserves $U(1)_{A}$ symmetry, acts only to mix $\eta^{\prime}$ and $\tilde{\eta}^{\prime}$.

In case (c), the non-zero quark mass increases both the $\eta_{1}^{\prime}$ and $\eta_{2}^{\prime}$ masses by

$$
m_{\eta_{1,2}^{\prime}}^{2}=m_{\eta_{1,2}^{\prime}}^{2}\left(m_{q}=0\right)+12\left(x_{3} \mp \frac{x_{1} x_{3}-x_{4}}{\sqrt{x_{1}^{2}-x_{2}}}\right) m_{q},
$$

with

$$
\begin{aligned}
& x_{3}=\frac{A_{0} \sigma}{f_{\eta^{\prime}}^{2}}+\frac{4 \Gamma_{1} d^{2}}{f_{\tilde{\eta}^{\prime}}^{2}}, \\
& x_{4}=2 \frac{6 c_{0} \Gamma_{1} d^{2} \sigma^{3}+4 \Gamma_{1} d^{2}\left(\gamma_{1} d^{2} \sigma+\lambda_{3} d^{2} \sigma^{2}\right)+A_{0} \sigma\left(4 \gamma_{1} d^{2} \sigma+\lambda_{3} d^{2} \sigma^{2}\right)}{f_{\eta^{\prime}}^{2} f_{\tilde{\eta}^{\prime}}^{2}} .
\end{aligned}
$$

For small $m_{q}, \tilde{\eta}_{1}^{\prime}$ becomes heavy owing to the $\sigma$-d coupling. By contrast, the pion $\pi_{1}$ remains light, even in the presence of the coupling.

[1] Y. Nambu and G. Jona-Lasinio, Phys. Rev. 124, 246 (1961); ibid. 122345 (1961).

[2] Reviewed in T. Hatsuda and T. Kunihiro, Phys. Rept. 247, 221 (1994).

[3] J.C. Collins and M.J. Perry, Phys. Rev. Lett. 34, 1353 (1975); G. Baym and S.A. Chin, Phys. Lett. B62, 241 (1976).

[4] B. Barrois, Nucl. Phys. B129, 390 (1977); D. Bailin and A. Love, Phys. Rept. 107, 325 (1984); M. Iwasaki and T. Iwado, Phys. Lett. B350, 163 (1995); R. Rapp, T. Schäfer, E. V. Shuryak and M. Velkovsky, Phys. Rev. Lett. 81, 53 (1998); M. G. Alford, K. Rajagopal and F. Wilczek, Phys. Lett. B422, 247 (1998).

[5] Reviewed in K. Rajagopal and F. Wilczek, in Handbook of QCD, ed. M. Shifman (World Scientific, Singapore, 2001); M.G. Alford, Ann. Rev. Nucl. Part. Sci. 51, 131 (2001).

[6] K. Yagi, T. Hatsuda and Y. Miake, Quark-Gluon Plasma, Cambridge Univ. Press (Cambridge, 2005).

[7] M. Sigrist and K. Ueda, Rev. Mod. Phys. 63, 239 (1991).

[8] Reviewed in M. Buballa, Phys. Rept. 407, 205 (2005).

[9] Reviewed in B. Vanderheyden and A.D. Jackson, AIP Conf. Proc. 775, 220 (2005) hep-ph/0503032.

[10] T. Hatsuda, M. Tachibana, N. Yamamoto and G. Baym, Phys. Rev. Lett 97, 122001 (2006).

[11] T. Schäfer and F. Wilczek, Phys. Rev. Lett. 82, 3956 (1999).

[12] R.D. Pisarski and F. Wilczek, Phys. Rev. D29, 338 (1984).

[13] K. Iida and G. Baym, Phys. Rev. D 63, 074018 (2001); ibid. 66, 059903(E) (2002).

[14] I. Giannakis and H.-c. Ren, Nucl. Phys. B 669, 462 (2003).

[15] K. Iida, T. Matsuura, M. Tachibana and T. Hatsuda, Phys. Rev. D 71, 054003 (2005).

[16] D. T. Son and M. A. Stephanov, Phys. Rev. D 61, 074012 (2000).

[17] T. Schäfer, Phys. Rev. D 65, 094033 (2002). R. Rapp, T. Schäfer, E. V. Shuryak and M. Velkovsky, Annals Phys. 280, 35 (2000).

[18] M. Asakawa and K. Yazaki, Nucl. Phys. A 504, 668 (1989). A. Barducci, R. Casalbuoni, S. De Curtis, R. Gatto and G. Pettini, Phys. Lett. B 231, 463 (1989). M. Stephanov, K. Rajagopal and E. Shuryak, Phys. Rev. Lett. 81, 4816 (1998). Y. Hatta and T. Ikeda, Phys. Rev. D 67, 014028 (2003).

[19] T. Matsuura, K. Iida, T. Hatsuda, and G. Baym, Phys. Rev. D 69, 074012 (2004). I. Giannakis, D.-f. Hou, H.-c. Ren and D. H. Rischke, Phys. Rev. Lett. 93, 232301 (2004).

[20] P. M. Chaikin and T. C. Lubensky, Principles of Condensed Matter Physics, Cambridge Univ. Press (Cambridge, 1995), Ch. 4.6.4.

[21] B. Vanderheyden and A. D. Jackson, Phys. Rev. D 62, 094010 (2000).

[22] M. Kitazawa, T. Koide, T. Kunihiro and Y. Nemoto, Prog. Theor. Phys. 108, 929 (2002); ibid. 110, 185 (2003).

[23] K. Fukushima, Phys. Rev. D 70, 094014 (2004).

[24] R. Casalbuoni and R. Gatto, Phys. Lett. B 464, 111 (1999).

[25] M. Rho, A. Wirzba and I. Zahed, Phys. Lett. B473, 126 (2000).

[26] A. H. Fariborz, R. Jora and J. Schechter, hep-ph/0612200

[27] J. Gasser and H. Leutwyler, Nucl. Phys. B 250, 465 (1985). 
[28] D. K. Hong, T. Lee and D. P. Min, Phys. Lett. B 477, 137 (2000). S. R. Beane, P. F. Bedaque and M. J. Savage, Phys. Lett. B 483, 131 (2000).

[29] C. Manuel and M. H. G. Tytgat, Phys. Lett. B 479, 190 (2000). T. Schäfer, Phys. Rev. D 65, 074006 (2002).

[30] P. F. Bedaque and T. Schäfer, Nucl. Phys. A 697, 802 (2002).

[31] M.G. Alford, K. Rajagopal, and F. Wilczek, Nucl. Phys. B 537, 443 (1999). 